(C) [2005] IEEE. Reprinted, with permission, from [Shoudong Huang, A unified approach to controller design for achieving ISS and related properties, Automatic Control, IEEE Transactions on (Volume:50, Issue: 11), 14 November 2005]. This material is posted here with permission of the IEEE. Such permission of the IEEE does not in any way imply IEEE endorsement of any of the University of Technology, Sydney's products or services. Internal or personal use of this material is permitted. However, permission to reprint/republish this material for advertising or promotional purposes or for creating new collective works for resale or redistribution must be obtained from the IEEE by writing to pubs-permissions@ieee.org. By choosing to view this document, you agree to all provisions of the copyright laws protecting it 


\title{
A Unified Approach to Controller Design for Achieving ISS and Related Properties*
}

\author{
Shoudong Huang ${ }^{\dagger} \quad$ M.R. James ${ }^{\ddagger} \quad$ Dragan Nešic $^{\S} \quad$ Peter Dower
}

March 16, 2005

\begin{abstract}
A unified approach to the design of controllers achieving various specified inputto-state stability (ISS) like properties is presented. Both full state and measurement feedback cases are considered. Synthesis procedures based on dynamic programming is given using the recently developed results on controller synthesis to achieve uniform $l^{\infty}$ bound [12]. Our results make an important connection between the ISS literature and nonlinear $H^{\infty}$ design methods.
\end{abstract}

\section{Introduction}

Analysis and design of control systems with disturbances is one of the central topics in control engineering that is continuing to attract a lot of research interest in the context of nonlinear systems. This trend has been driven by several major breakthroughs over the past 15 years that occurred in nonlinear $H^{\infty}$ control (e.g. $\left.[3,4,26,11]\right)$ and the input to state stability (ISS) related literature (e.g. [24, 21, 2]). These two approaches have been developed relatively independently of each other and they differ in stability properties that are considered, tools that are used and questions that are asked. Both approaches have their advantages and disadvantages but they both provide invaluable tools and insight into the problems of analysis and design of nonlinear control systems with disturbances.

Nonlinear $H^{\infty}$ control has its roots in the theory of linear $H^{\infty}$ control (from which the name is inherited). The main objective of research in nonlinear $H^{\infty}$ control has been

*This work was supported by the Australian Research Council.

${ }^{\dagger}$ Centre of Excellence in Autonomous Systems, Faculty of Engineering, The University of Technology, Sydney, PO Box 123, Broadway, NSW 2007, Australia.shoudong.huang@uts.edu.au

${ }^{\ddagger}$ Department of Engineering, Australian National University, Canberra, ACT 0200, Australia. Matthew.James@anu.edu.au

${ }^{\S}$ Department of Electrical and Electronic Engineering, The University of Melbourne, Parkville, 3010, Victoria, Australia.d.nesic@ee.mu.oz.au

IDepartment of Electrical and Electronic Engineering, The University of Melbourne, Parkville, 3010, Victoria, Australia. p.dower@ee.mu.oz.au 
to develop analysis and design tools to achieve controllers robust against uncertainty. The framework and tools used to solve the nonlinear problem originate from optimal control (including risk-sensitive stochastic optimal control), game theory, and dissipative systems. Dynamic programming is a key technique in all these areas. Willems' theory [27] of dissipative systems is an elegant and powerful technique for stability analysis with strong links to Lyapunov stability theory (storage functions play the role of Lyapunov functions).

Research in nonlinear $H^{\infty}$ control has proceeded to date to translate linear $H^{\infty}$ control results to a nonlinear setting to the extent possible. In this context, it is typical to model the plant and controller as nonlinear operators and to consider $L^{2}$ stability with a finite (linear) gain of the closed loop system, which comes from its linear tradition. Moreover, this literature often aims at designing controllers that achieve minimum (optimal), or near minimum, gains from disturbance inputs to plant outputs and, hence, controller design often requires a solution of an appropriate dynamic programming equation (DPE) or inequality (DPI). An advantage of this approach is that it can be applied to a very broad class of plants and its main drawback is the heavy computation required to solve DPE/DPI [11]. Nevertheless, the methodology is fundamental and provides useful conceptual insights. Note that while much of the existing literature has focused on linear gains, the tools and techniques used apply much more generally, as we shall see.

The ISS related literature builds on the tradition of stability of dynamical systems and Lyapunov theory. Research in this area has concentrated on finding appropriate nonlinear generalizations of different finite gain input-output stability properties that are more natural in the nonlinear context and fully compatible with Lyapunov theory. The plant is modelled as a dynamical system with disturbance inputs and the related stability properties usually make use of nonlinear gains. The majority of ISS related research has concentrated on presenting different equivalent characterizations of ISS like properties $[23,24,10,2]$, proving appropriate small gains theorems [13] and applying the ISS like properties to analysis and controller design. This literature is usually not concerned with computing minimum disturbance gains and the main tool for applying these results are Lyapunov like functions that are very difficult to find. Typically, abstract existence results are used, or else explicit constructions for special classes of systems. We are not aware of any results that provide a systematic procedure for controller design for general nonlinear systems that achieves different ISS like stability properties for the plant dynamics.

The purpose of this paper is to exploit techniques typically used in nonlinear $H^{\infty}$ control to address the problem of controller design with the goal of achieving different ISS like properties for the plant dynamics. In particular, we use recent results on uniform $L^{\infty}$ bounded (ULIB) robustness [12] that employ nonlinear dissipative systems and $H^{\infty}$ techniques in an appropriate $L^{\infty}$ stability setting. Our main results show that a range of controller design problems achieving appropriate ISS like properties for the plant dynamics can be solved by solving another ULIB problem for an auxiliary augmented plant. We present our results in a unifying manner to show that controllers achieving any of the following properties can be designed via appropriate ULIB problems: input to state stability (ISS) [19], integral input to state stability (iISS) [2], integral input to integral state stability (iIiSS) [20], input to output stability (IOS) [25], input output to state stability (IOSS) [16] and incremental input to state stability ( $\delta$ ISS) [1]. It will become apparent 
that further ISS like properties can be achieved using the same technique. Important features of our approach are: (i) we need to fix the desired disturbances gains and transients bounds prior to controller design; (ii) admissible controllers we consider are causal operators and our solutions can be interpreted as a dynamical controller with an appropriate initialization; (iii) we achieve an ISS like bound only for the plant dynamics and controller dynamics is not considered; (iv) we consider both full state and measurement feedback problems; (v) our controllers are obtained via solutions of appropriate DPE/DPI and in general they are computationally very demanding.

In the synthesis problems in this paper, we consider only plant states in the inputoutput bounds. This mainly because that the initial condition of controller are easily be set in contrast to plant initial states. We show (in Section 8.1) that certain robustness with respect to the controller initialization can be achieved by our design. Moreover, our approach is flexible and it may be modified so that we actually achieve input-output bounds for the closed-loop system including controller state (see Section 8.2), but then it is harder to have clear statements of the main results. Some detailed discussion on this issue are given in Remark 4.4 .

In this paper the results have been presented for discrete time systems. The continuous time case is more technical and will be considered in our future research work. This paper is organized as follows. Preliminaries and notations are given in Section 2. In Section 3, we present a unified definition for 6 different ISS like properties. In Section 4, we state the state feedback and measurement feedback synthesis problems considered in this paper. The problems are then transferred into ULIB synthesis problems in Section 5. In Section 6 , the dynamic programming results are presented using the existing ULIB results. A simple illustrate example is given in Section 7. Some further remarks on our method are presented in Section 8. Section 9 concludes the paper.

\section{Preliminaries}

Sets of real numbers, nonnegative real numbers, integers and nonnegative integers are denoted respectively as $\mathbf{R}, \mathbf{R}_{+}, \mathbf{Z}$ and $\mathbf{Z}_{+}$. Moreover, we denote

$$
\overline{\mathbf{R}}:=\mathbf{R} \cup\{+\infty\}, \quad \tilde{\mathbf{R}}:=\mathbf{R} \cup\{+\infty\} \cup\{-\infty\} .
$$

Recall that a function $\gamma: \mathbf{R}_{+} \rightarrow \mathbf{R}_{+}$is of class $\mathcal{K}$ if it is continuous, strictly increasing and $\gamma(0)=0$; it is of class $\mathcal{K}_{\infty}$ if it is of class $\mathcal{K}$ and also $\gamma(s) \rightarrow \infty$ as $s \rightarrow \infty$. A function $\beta: \mathbf{R}_{+} \times \mathbf{R}_{+} \rightarrow \mathbf{R}_{+}$is said to be a function of class $\mathcal{K} \mathcal{L}$ if for each fixed $t \geq 0, \beta(\cdot, t)$ is of class $\mathcal{K}$ and for each fixed $s \geq 0, \beta(s, \cdot)$ decreases to zero.

Sontag [20] proved the following lemma on $\mathcal{K} \mathcal{L}$ functions that we need.

Lemma 2.1 [20] Given arbitrary $\beta \in \mathcal{K} \mathcal{L}$, there exist two functions $\alpha_{1}, \alpha_{2} \in \mathcal{K}_{\infty}$ such that

$$
\beta(s, t) \leq \beta_{1}(s, t)=\alpha_{1}\left(\alpha_{2}(s) e^{-t}\right), \quad \forall s \geq 0, t \geq 0
$$


Given $\mathbf{W} \subseteq \mathbf{R}^{s}, \forall k \in \mathbf{Z}_{+}$, we use the following notation:

$$
\begin{aligned}
w_{[0, k-1]} & :=\left\{w_{0}, \cdots, w_{k-1}\right\}, \forall k \geq 0, \\
\mathcal{W}_{[0, k-1]} & :=\left\{w_{[0, k-1]}: w_{i} \in \mathbf{W}, 0 \leq i \leq k-1\right\}, \\
\mathcal{W}_{[0, \infty)} & :=\left\{w_{[0, \infty)}: w_{i} \in \mathbf{W}\right\}
\end{aligned}
$$

Sometimes we use the notation $w=w_{[0, \infty)}$. We use the convention that $\mathcal{W}_{[0,-1]}=\emptyset$. In the sequel, we use the notation $x_{[0, k]}, \mathcal{X}_{[0, k]}, \mathcal{X}_{[0, \infty)}, \mathcal{U}_{[0, \infty)}, y_{[0, k-1]}, \mathcal{Y}_{[0, k-1]}, \mathcal{Y}_{[0, \infty)}$, etc, which have meanings analogous to (3). We also use the following notation:

$$
\left\|w_{[0, k-1]}\right\|_{\infty}:=\max _{0 \leq i \leq k-1}\left|w_{i}\right|
$$

where $|\cdot|$ is the Euclidean norm. To simplify the notation, for any two vectors $x_{1}$ and $x_{2}$, sometimes we also denote $\left(x_{1}^{T} x_{2}^{T}\right)^{T}$ as $\left(x_{1}, x_{2}\right)$.

\section{A unified definition for ISS like properties}

One aspect of our contribution is a unified approach to solving a range of control design problems that achieve various Input-to-State Stability (ISS) like properties for the plant in the closed loop system. The first step in this unified approach is to provide a unified definition of a range of ISS like properties that have been considered recently in the literature. In this section we first define a range of seemingly unrelated ISS like properties in Definition 3.1 and then in Definition 3.4 we restate all in a unified and compact manner that is particularly suited for our approach.

Consider the following system with input sequence $\left\{w_{k}\right\}$ and output sequence $\left\{\bar{z}_{k}\right\}$

$$
\begin{aligned}
x_{k+1} & =f\left(x_{k}, w_{k}\right) \\
\bar{z}_{k} & =H\left(x_{k}\right)
\end{aligned}
$$

where $x_{k} \in \mathbf{R}^{n}, w_{k} \in \mathbf{W} \subseteq \mathbf{R}^{s}, \bar{z}_{k} \in \mathbf{R}^{q}$. We denote by $\phi\left(k, x_{0}, w_{[0, k-1]}\right)$ the solution of the system at time $k$ that starts from the initial condition $x_{0}$ and under the action of the input $w_{[0, k-1]}$. Sometimes we simply use $\phi_{k}$ or $x_{k}$ to denote $\phi\left(k, x_{0}, w_{[0, k-1]}\right)$. A range of ISS like properties that have been introduced in the literature $[19,14,2,20,25,16,1]$ are listed below:

Definition 3.1 Let $B_{0} \subseteq \mathbf{R}^{n}, \mathbf{W} \subseteq \mathbf{R}^{s}$, the system (4) is:

- Input to State Stable (ISS) [19, 14] if there exist $\beta \in \mathcal{K} \mathcal{L}$ and $\gamma_{1} \in \mathcal{K}$ such that the trajectories of the system satisfy:

$$
\left|\phi\left(k, x_{0}, w_{[0, k-1]}\right)\right| \leq \beta\left(\left|x_{0}\right|, k\right)+\gamma_{1}\left(\left\|w_{[0, k-1]}\right\|_{\infty}\right),
$$

for all $x_{0} \in B_{0}, w_{[0, k-1]} \in \mathcal{W}_{[0, k-1]}$, and $k \geq 0$. 
- Integral Input to State Stable (iISS)[2] if there exist $\beta \in \mathcal{K} \mathcal{L}$ and $\gamma_{1}, \gamma_{2} \in \mathcal{K}$ such that:

$$
\left|\phi\left(k, x_{0}, w_{[0, k-1]}\right)\right| \leq \beta\left(\left|x_{0}\right|, k\right)+\gamma_{1}\left(\sum_{i=0}^{k-1} \gamma_{2}\left(\left|w_{i}\right|\right)\right),
$$

for all $x_{0} \in B_{0}, w_{[0, k-1]} \in \mathcal{W}_{[0, k-1]}$, and $k \geq 0$.

- Integral Input to Integral State Stable (iIiSS)[20] if there exist $\gamma_{i} \in \mathcal{K}, i=1,2, \ldots, 5$ such that:

$$
\gamma_{1}\left(\sum_{i=0}^{k-1} \gamma_{2}\left(\left|\phi\left(i, x_{0}, w_{[0, i-1]}\right)\right|\right)\right) \leq \gamma_{3}\left(\left|x_{0}\right|\right)+\gamma_{4}\left(\sum_{i=0}^{k-1} \gamma_{5}\left(\left|w_{i}\right|\right)\right)
$$

for all $x_{0} \in B_{0}, w_{[0, k-1]} \in \mathcal{W}_{[0, k-1]}$, and $k \geq 0$.

- Input to Output Stable (IOS)[25] if there exist $\beta \in \mathcal{K} \mathcal{L}$ and $\gamma_{1} \in \mathcal{K}$ such that:

$$
\left|H\left(\phi\left(k, x_{0}, w_{[0, k-1]}\right)\right)\right| \leq \beta\left(\left|x_{0}\right|, k\right)+\gamma_{1}\left(\left\|w_{[0, k-1]}\right\|_{\infty}\right),
$$

for all $x_{0} \in B_{0}, w_{[0, k-1]} \in \mathcal{W}_{[0, k-1]}$, and $k \geq 0$.

- Input Output to State Stable (IOSS)[16] if there exist $\beta \in \mathcal{K} \mathcal{L}$ and $\gamma_{1}, \gamma_{2} \in \mathcal{K}$ such that:

$$
\left|\phi\left(k, x_{0}, w_{[0, k-1]}\right)\right| \leq \beta\left(\left|x_{0}\right|, k\right)+\gamma_{1}\left(\left\|w_{[0, k-1]}\right\|_{\infty}\right)+\gamma_{2}\left(\left\|z_{[0, k-1]}\right\|_{\infty}\right),
$$

for all $x_{0} \in B_{0}, w_{[0, k-1]} \in \mathcal{W}_{[0, k-1]}$, and $k \geq 0$.

- Incrementally Input to State Stable ( $\delta I S S)[1]$ if there exist $\beta \in \mathcal{K} \mathcal{L}$ and $\gamma_{1} \in \mathcal{K}$ such that:

$$
\left|\phi\left(k, x_{0}^{1}, w_{[0, k-1]}^{1}\right)-\phi\left(k, x_{0}^{2}, w_{[0, k-1]}^{2}\right)\right| \leq \beta\left(\left|x_{0}^{1}-x_{0}^{2}\right|, k\right)+\gamma_{1}\left(\left\|\left(w^{1}-w^{2}\right)_{[0, k-1]}\right\|_{\infty}\right),
$$

for all $x_{0}^{1}, x_{0}^{2} \in B_{0}, w_{[0, k-1]}^{1}, w_{[0, k-1]}^{2} \in \mathcal{W}_{[0, k-1]}$, and $k \geq 0$.

Remark 3.2 The original ISS-like properties are usually defined globally. That is in Definition $3.1 B_{0}=\mathbf{R}^{n}, \mathbf{W}=\mathbf{R}^{s}$. However, similar properties can be introduced for more general sets $B_{0} \subseteq \mathbf{R}^{n}, \mathbf{W} \subseteq \mathbf{R}^{s}$. In this paper, we will consider this general case. Our methods are also applicable to the more general practical ISS-like properties (e.g. [13]) but we are not addressing them here.

Remark 3.3 By Lemma 2.1, any $\beta \in \mathcal{K} \mathcal{L}$ has an upper bound of the form $\beta_{1}(s, t)=$ $\alpha_{1}\left(\alpha_{2}(s) e^{-t}\right)$. Notice that $\beta_{1}$ itself is also a $\mathcal{K} \mathcal{L}$ function, so the properties in Definition 3.1 are qualitatively equivalent to the properties where the function $\beta(\cdot, k)$ is replaced by $\alpha_{1}\left(\alpha_{2}(\cdot) e^{-k}\right)$. For example, system (4) is ISS if and only if there exist $\alpha_{1}, \alpha_{2} \in \mathcal{K}_{\infty}$ and $\gamma_{1} \in \mathcal{K}$ such that the trajectories of (4) satisfy:

$$
\left|\phi\left(k, x_{0}, w_{[0, k-1]}\right)\right| \leq \alpha_{1}\left(\alpha_{2}\left(\left|x_{0}\right|\right) e^{-k}\right)+\gamma_{1}\left(\left\|w_{[0, k-1]}\right\|_{\infty}\right),
$$

for all $x_{0} \in B_{0}, w_{[0, k-1]} \in \mathcal{W}_{[0, k-1]}$, and $k \geq 0$. Certainly, the bound $\alpha_{1}\left(\alpha_{2}\left(\left|x_{0}\right|\right) e^{-k}\right)$ may be not as tight as the bound $\beta\left(\left|x_{0}\right|, k\right)$ with $\beta \in \mathcal{K} \mathcal{L}$. In this paper, we will only consider the case when $\mathcal{K} \mathcal{L}$ function is of the form $\alpha_{1}\left(\alpha_{2}(s) e^{-t}\right)$. 


\begin{tabular}{|c|c|c|c|c|c|}
\hline $\begin{array}{c}\text { Property } \\
\star\end{array}$ & $\rho^{\star}\left(x_{0}, k\right)$ & $\psi_{k}^{\star}\left(w_{[0, k-1]}\right)$ & $\varphi_{k}^{\star}\left(\phi_{[0, k-1]}\right)$ & $G^{\star}(\phi, \rho, \psi, \varphi)$ & $n^{\star}$ \\
\hline ISS & $\alpha_{2}\left(\left|x_{0}\right|\right) e^{-k}$ & $\left\|w_{[0, k-1]}\right\|_{\infty}$ & 0 & $|\phi|-\alpha_{1}(\rho)-\gamma_{1}(\psi)$ & $n+2$ \\
\hline iISS & $\alpha_{2}\left(\left|x_{0}\right|\right) e^{-k}$ & $\sum_{i=0}^{k-1} \gamma_{2}\left(\left|w_{i}\right|\right)$ & 0 & $|\phi|-\alpha_{1}(\rho)-\gamma_{1}(\psi)$ & $n+2$ \\
\hline iIiSS & $\gamma_{3}\left(\left|x_{0}\right|\right)$ & $\sum_{i=0}^{k-1} \gamma_{5}\left(\left|w_{i}\right|\right)$ & $\sum_{i=0}^{k-1} \gamma_{2}\left(\left|\phi_{i}\right|\right)$ & $\gamma_{1}(\varphi)-\rho-\gamma_{4}(\psi)$ & $n+3$ \\
\hline IOS & $\alpha_{2}\left(\left|x_{0}\right|\right) e^{-k}$ & $\left\|w_{[0, k-1]}\right\|_{\infty}$ & 0 & $|H(\phi)|-\alpha_{1}(\rho)-\gamma_{1}(\psi)$ & $n+2$ \\
\hline $\operatorname{IOSS}^{1}$ & $\alpha_{2}\left(\left|x_{0}\right|\right) e^{-k}$ & $\left\|w_{[0, k-1]}\right\|_{\infty}$ & $\left\|H(\phi)_{[0, k-1]}\right\|_{\infty}$ & $|\phi|-\alpha_{1}(\rho)-\gamma_{1}(\psi)-\gamma_{2}(\varphi)$ & $n+3$ \\
\hline$\delta \mathrm{ISS}^{2}$ & $\alpha_{2}\left(\left|x_{0}^{1}-x_{0}^{2}\right|\right) e^{-k}$ & $\left\|\left(w^{1}-w^{2}\right)_{[0, k-1]}\right\|_{\infty}$ & 0 & $\left|\phi^{1}-\phi^{2}\right|-\alpha_{1}(\rho)-\gamma_{1}(\psi)$ & $n+2$ \\
\hline
\end{tabular}

Table 1: Summary of the data needed in the unifying definition for ISS like properties (equation (6))

We find it useful to restate Definition 3.1 since its new form is more suited for our paper. First, note that the inequality (5) in the ISS definition is:

$$
\left|\phi\left(k, x_{0}, w_{[0, k-1]}\right)\right|-\alpha_{1}\left(\alpha_{2}\left(\left|x_{0}\right|\right) e^{-k}\right)-\gamma_{1}\left(\left\|w_{[0, k-1]}\right\|_{\infty}\right) \leq 0 .
$$

Hence, the definition can be restated as follows. There exists mappings $G^{I S S}: \mathbf{R}^{n} \times$ $\mathbf{R} \times \mathbf{R} \rightarrow \mathbf{R}, \rho^{I S S}: \mathbf{R}^{n} \times \mathbf{Z}_{+} \rightarrow \mathbf{R}_{+}$and for every $k \in \mathbf{Z}_{+}$there exists a mapping $\psi_{k}^{I S S}: \mathcal{W}_{[0, k-1]} \rightarrow \mathbf{R}_{+}$, such that

$$
G^{I S S}\left(\phi\left(k, x_{0}, w_{[0, k-1]}\right), \rho^{I S S}\left(x_{0}, k\right), \psi_{k}^{I S S}\left(w_{[0, k-1]}\right)\right) \leq 0,
$$

for all $x_{0} \in B_{0}, w_{[0, k-1]} \in \mathcal{W}_{[0, k-1]}$, and $k \geq 0$. In fact, we have that

$$
\begin{aligned}
\rho^{I S S}\left(x_{0}, k\right) & :=\alpha_{2}\left(\left|x_{0}\right|\right) e^{-k} \\
\psi_{k}^{I S S}\left(w_{[0, k-1]}\right) & :=\left\|w_{[0, k-1]}\right\|_{\infty}, \\
G^{I S S}(\phi, \rho, \psi) & :=|\phi|-\alpha_{1}(\rho)-\gamma_{1}(\psi),
\end{aligned}
$$

where $\gamma_{1} \in \mathcal{K}$ and $\alpha_{1}, \alpha_{2} \in \mathcal{K}_{\infty}$. We use the convention that $\psi_{k}^{I S S}(\emptyset)=0$ and note that since $w_{[0,-1]}=\emptyset$, we have that $\psi_{0}^{I S S}\left(w_{[0,-1]}\right)=0$.

Using the same arguments as above, we can restate each property in Definition 3.1 in the same manner. The summary of all situations is presented in Table 1 that is used in conjunction with the following:

Definition 3.4 [Unified definition for ISS like properties] Let $B_{0} \subseteq \mathbf{R}^{n}$ and $\mathbf{W} \subseteq \mathbf{R}^{s}$ be given. The system (4) has Property $\star$, where $\star \in\{I S S, i I S S, i I i S S, I O S, I O S S, \delta I S S\}$, if there exist $\gamma_{i} \in \mathcal{K}, i=1,2, \ldots, 5$ and $\alpha_{1}, \alpha_{2} \in \mathcal{K}_{\infty}$ such that with $\rho^{\star}(\cdot, \cdot), \psi_{k}^{\star}(\cdot), \varphi_{k}^{\star}(\cdot)$ and $G^{\star}(\cdot, \cdot, \cdot, \cdot)$ defined in Table 1 we have that the solutions of the system (4) satisfy:

$$
G^{\star}\left(\phi\left(k, x_{0}, w_{[0, k-1]}\right), \rho^{\star}\left(x_{0}, k\right), \psi_{k}^{\star}\left(w_{[0, k-1]}\right), \varphi_{k}^{\star}\left(\phi_{[0, k-1]}\right)\right) \leq 0,
$$

\footnotetext{
${ }^{1}$ We use the notation $H(\phi)_{[0, k-1]}$ to denote the sequence $H\left(\phi\left(i, x_{0}, w_{[0, i-1]}\right)\right), i=0,1, \ldots, k-1$.

${ }^{2}$ The meaning of notation used in this row of the table is explained in Remark 3.5.
} 
for all $x_{0} \in B_{0}, w_{[0, k-1]} \in \mathcal{W}_{[0, k-1]}, k \geq 0$, where $\phi_{[0, k-1]}$ denotes the sequence of solutions $\phi\left(i, x_{0}, w_{[0, i-1]}\right), i=0,1, \ldots, k-1$ of the system (4).

Remark 3.5 Note that the $\delta$ ISS property for the system

$$
\tilde{x}_{k+1}=\tilde{f}\left(\tilde{x}_{k}, \tilde{w}_{k}\right)
$$

can be investigated via an augmented auxiliary system of the form:

$$
\begin{aligned}
& \tilde{x}_{k+1}^{1}=\tilde{f}\left(\tilde{x}_{k}^{1}, \tilde{w}_{k}^{1}\right), \\
& \tilde{x}_{k+1}^{2}=\tilde{f}\left(\tilde{x}_{k}^{2}, \tilde{w}_{k}^{2}\right),
\end{aligned}
$$

which consists of two exact copies of the original system that are initialized respectively from the initial conditions $\tilde{x}_{0}^{1}$ and $\tilde{x}_{0}^{2}$ and that are driven with the inputs $\tilde{w}^{1}$ and $\tilde{w}^{2}$. We can say that the system (8) has the form (4) if we define

$$
x:=\left(\begin{array}{c}
\tilde{x}^{1} \\
\tilde{x}^{2}
\end{array}\right) ; \quad w:=\left(\begin{array}{c}
\tilde{w}^{1} \\
\tilde{w}^{2}
\end{array}\right) ; \quad f(x, w):=\left(\begin{array}{c}
\tilde{f}\left(\tilde{x}^{1}, \tilde{w}^{1}\right) \\
\tilde{f}\left(\tilde{x}^{2}, \tilde{w}^{2}\right)
\end{array}\right) .
$$

In the sequel, whenever we talk about $\delta$ ISS of the system (4), we will always assume that the above given transformation has already been carried out and hence the system has the form (8) with (9) (we assume that the dimensions $n$ and $s$ of $x$ and $w$ respectively are even). And we actually mean the $\delta$ ISS property of the system (7) (where $\tilde{x}$ and $\tilde{w}$ respectively have a dimension $n / 2$ and $s / 2$ ).

Remark 3.6 There are two reasons for restating Definition 3.1 as in Definition 3.4. First, the inequality (6) will be shown to be related to an inequality in the Uniform $l^{\infty}$ Boundedness (ULIB) problem that was recently considered and solved in the literature [12]. Moreover, we will show how to transform our problem that involves some of the properties in Definition 3.1 into an auxiliary ULIB problem that can be solved using techniques of [12]. The inequality (6) is especially suited for this problem transformation. Second, our results are unifying for all ISS like properties of Definition 3.1 and, hence, Definition 3.4 provides a compact way of presenting our proofs and results.

Remark 3.7 A range of other stability and detectability properties can be captured by using the same Definition 3.4 and augmenting the Table 1 in an appropriate manner by specifying $\rho^{\star}(\cdot, \cdot), \psi_{k}^{\star}(\cdot), \varphi_{k}^{\star}(\cdot), G^{\star}(\cdot, \cdot, \cdot, \cdot)$ for the new properties. We have not exhausted all possible candidate properties in Table 1 , but rather concentrated on the most representative properties that were considered in the literature.

\section{Problem Statements}

In this section we pose several controller design problems. First, we state the full state feedback controller design problem with the goal of achieving one of the properties from Table 1 for the plant state in the closed loop system. Second, we state the measurement 
feedback problem that achieves one of the properties from Table 1 for the plant state in the closed loop system. We will solve these two problems by transforming them into two auxiliary problems (full state feedback and measurement feedback ULIB problems) that were recently considered and solved in the literature (see [12]). In this section we also provide definitions of the ULIB problems.

For the full state feedback case, consider the nonlinear discrete-time system

$$
\begin{aligned}
x_{k+1} & =f\left(x_{k}, u_{k}, w_{k}\right), \quad k \geq 0, \\
\bar{z}_{k} & =H\left(x_{k}\right), k \geq 0 .
\end{aligned}
$$

Here $x_{k} \in \mathbf{R}^{n}, \bar{z}_{k} \in \mathbf{R}^{q}, u_{k} \in \mathbf{U} \subseteq \mathbf{R}^{m}$ and $w_{k} \in \mathbf{W} \subseteq \mathbf{R}^{s}$ are the state, output, control input and disturbance input, respectively.

Before stating the problems of interest, we define the class of admissible controllers that our designs will yield. For system (10), let $X=\mathbf{R}^{n}$ and $\mathbf{U} \subseteq \mathbf{R}^{m}$ be given, define $\mathcal{X}_{[0, \infty)}$ and $\mathcal{U}_{[0, \infty)}$ similarly as in (3). An admissible state feedback controller is a causal map $K: \mathcal{X}_{[0, \infty)} \rightarrow \mathcal{U}_{[0, \infty)}$, meaning that for each time $k \geq 0$ if $x^{1}, x^{2} \in \mathcal{X}_{[0, \infty)}$ and $x_{l}^{1}=x_{l}^{2}$ for all $0 \leq l \leq k$ then $K\left(x^{1}\right)_{k}=K\left(x^{2}\right)_{k}$. i.e. the control at any time $k$ is independent of the future states. We denote the set of admissible state feedback controllers as

$$
\mathcal{C}_{s f}:=\left\{K: \mathcal{X}_{[0, \infty)} \rightarrow \mathcal{U}_{[0, \infty)}, K \text { is causal }\right\}
$$

We sometimes abuse the notation by writing $u_{k}=K\left(x_{[0, k]}\right)$. Also, the state trajectories of the plant in the closed loop system consisting of the system (10) and a given admissible controller $u_{k}=K\left(x_{[0, k]}\right)$ are denoted as $\phi\left(k, x_{0}, u, w_{[0, k-1]}\right)$. Note that the class of admissible controllers is very large and it includes static and dynamic controllers, as well as a number of other configurations.

The first problem that we consider is stated next. This problem is referred to as a State Feedback $\star$ Problem where $\star$ can be any property listed in Table 1.

State Feedback $\star(\mathbf{S F} \star)$ Problem: Consider system (10), let $B_{0} \subseteq \mathbf{R}^{n}, \mathbf{W} \subseteq \mathbf{R}^{s}, \gamma_{i} \in$ $\mathcal{K}, i=1,2, \ldots, 5, \alpha_{1}, \alpha_{2} \in \mathcal{K}_{\infty}$ and $\star \in\{I S S, i I S S, i I i S S, I O S, I O S S, \delta I S S\}$ be given and define the functions $\rho^{\star}(\cdot, \cdot), \psi_{k}^{\star}(\cdot), \varphi_{k}^{\star}(\cdot)$ and $G^{\star}(\cdot, \cdot, \cdot, \cdot)$ as generated by Table 1 . Find, if possible, an admissible state feedback controller $K \in \mathcal{C}_{s f}$ such that the trajectories of the plant in the closed loop system satisfy the following:

$$
G^{\star}\left(\phi\left(k, x_{0}, u, w_{[0, k-1]}\right), \rho^{\star}\left(x_{0}, k\right), \psi_{k}^{\star}\left(w_{[0, k-1]}\right), \varphi_{k}^{\star}\left(\phi_{[0, k-1]}\right)\right) \leq 0,
$$

for all $x_{0} \in B_{0}, w_{[0, k-1]} \in \mathcal{W}_{[0, k-1]}, k \geq 0$. Here we use $\phi_{[0, k-1]}$ to denote the sequence $\phi\left(i, x_{0}, u, w_{[0, i-1]}\right), i=0,1, \ldots, k-1$. When there exists such a controller, we say that the $\mathrm{SF} \star$ Problem is solvable for system (10).

Remark 4.1 In fact, the above definition can be regarded as 6 definitions. For example, when $\star=$ ISS, the problem is SFISS Problem.

Remark 4.2 Note a crucial difference between Definition 3.4 and the statement of the $\mathrm{SF} \star$ Problem. In the definition, we say that the property holds if there exist functions $\gamma_{i} \in$ 
$\mathcal{K}, i=1,2, \ldots, 5$ and $\alpha_{1}, \alpha_{2} \in \mathcal{K}_{\infty}$ such that an appropriate inequality holds. However, in the statement of the $\mathrm{SF} \star$ Problem we fix all the functions $\gamma_{i} \in \mathcal{K}, i=1,2, \ldots, 5$, $\alpha_{1}, \alpha_{2} \in \mathcal{K}_{\infty}$ and then attempt to find a controller that satisfies (12). Hence, if the controller does not exist for one set of $\gamma_{i} \in \mathcal{K}, i=1,2, \ldots, 5, \alpha_{1}, \alpha_{2} \in \mathcal{K}_{\infty}$, it may exist for another set of these functions. Obviously, this poses certain limitation in terms of how one can use our tools. However, our results are very useful in a range of engineering situations in which it makes sense to fix the gains prior to design. Moreover, our results can be used in an iterative manner, as in $H^{\infty}$ control, where, if a controller does not exist for a certain set of gains, we then increase the gains and then try to redesign the controller. Finding a design technique that does not require a priori fixing of the gain functions is highly desirable and is left for future research.

For the measurement feedback ISS like synthesis problem, consider the nonlinear discrete-time system

$$
\begin{aligned}
x_{k+1} & =f\left(x_{k}, u_{k}, w_{k}\right), \quad k \geq 0, \\
\bar{z}_{k} & =H\left(x_{k}\right), \quad k \geq 0, \\
y_{k} & =h\left(x_{k}, w_{k}\right), \quad k \geq 0
\end{aligned}
$$

Here $x_{k} \in \mathbf{R}^{n}, \bar{z}_{k} \in \mathbf{R}^{q}, u_{k} \in \mathbf{U} \subseteq \mathbf{R}^{m}, w_{k} \in \mathbf{W} \subseteq \mathbf{R}^{s}, y_{k} \in \mathbf{R}^{p}$ are the state, output, control input, disturbance input, and measured output, respectively.

Remark 4.3 Note that the measurement output $y$ in (13) is in general different from the output $\bar{z}=H(x)$ that is used to define the IOS and IOSS properties in Definition 3.1.

For system (13), let $\mathbf{Y}=$ range $\{h\} \subseteq \mathbf{R}^{p}$ and $\mathbf{U} \subseteq \mathbf{R}^{m}$ be given, define $\mathcal{Y}_{[0, \infty)}$ and $\mathcal{U}_{[0, \infty)}$ similarly as in (3). An admissible measurement feedback controller is a strictlycausal map $K: \mathcal{Y}_{[0, \infty)} \rightarrow \mathcal{U}_{[0, \infty)}$, meaning that for each time $k>0$ if $y^{1}, y^{2} \in \mathcal{Y}_{[0, \infty)}$ and $y_{l}^{1}=y_{l}^{2}$ for all $0 \leq l \leq k-1$ then $K\left(y^{1}\right)_{k}=K\left(y^{2}\right)_{k}$, i.e., the control at time $k$ is independent of current and future measurements. We denote the set of admissible measurement feedback controllers as

$$
\mathcal{C}_{m f}:=\left\{K: \mathcal{Y}_{[0, \infty)} \rightarrow \mathcal{U}_{[0, \infty)}, K \text { is causal }\right\}
$$

We sometimes abuse notation by writing $u_{k}=K\left(y_{[0, k-1]}\right)$. Also, we still denote the trajectories of the plant in the closed loop system consisting of the system (13) and a given admissible controller $u_{k}=K\left(y_{[0, k-1]}\right)$ as $\phi\left(k, x_{0}, u, w_{[0, k-1]}\right)$.

Measurement Feedback $\star(\mathbf{M F} \star)$ Problem: Consider system (13), let $B_{0} \subseteq \mathbf{R}^{n}, \mathbf{W} \subseteq$ $\mathbf{R}^{s}, \gamma_{i} \in \mathcal{K}, i=1,2, \cdots, 5, \alpha_{1}, \alpha_{2} \in \mathcal{K}_{\infty}$ and $\star \in\{I S S, i I S S$, iI $i S S$, IOS, IOSS, $\delta I S S\}$ be given and define the functions $\rho^{\star}(\cdot, \cdot), \psi_{k}^{\star}(\cdot), \varphi_{k}^{\star}(\cdot)$, and $G^{\star}(\cdot, \cdot, \cdot, \cdot)$ as generated by Table 1. Find, if possible, an admissible measurement feedback controller $K \in \mathcal{C}_{m f}$ such that the trajectories of the plant in the closed loop system satisfy (12). When there exists such a controller, we say that the MF $\star$ Problem is solvable for system (13).

Remark 4.4 The SF $\star$ and $\mathrm{MF} \star$ problems require only that a desired bound is achieved on the solutions of the plant whereas no such requirement is imposed on the states of a possibly dynamic controller. There are five reasons for this: (i) ISS-like properties for 
nonlinear systems provide a desired bound for any initial state of the system. However, for a closed-loop system, the initial state of the plant and the initial state of the controller play different roles. The initial state of the plant may be arbitrary. But the initial state of the controller can be chosen by the designer. Hence it may be too strong to require ISS-like bound to be obtained for any initial state of the plant and any initial state of the controller in the closed-loop system. (ii) We consider possibly dynamic feedback controller design where the dimension of the controller is not given before the design. (iii) As we will show in Section 8.1, the requirement (12) guarantees appropriate robustness to perturbation in the initialization of the controller. (iv) As we will show in Section 8.2 , the method proposed in this paper can be generalized to achieve closed-loop ISS-like properties, but it is hard to state clearly in a unified way what the achieved bounds are. (v) This requirement is compatible with definitions of nonlinear $H^{\infty}$ problems ([11]) and the ULIB problems that are stated next.

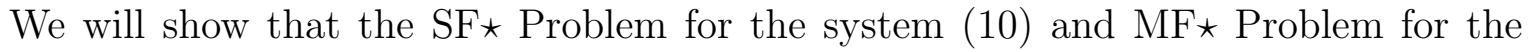
system (13) can be solved by solving the following controller synthesis problems for certain auxiliary systems. We first state the problems themselves and then introduce the auxiliary systems in the following section.

For the state feedback uniform $l^{\infty}$-bounded (ULIB) synthesis problem, we consider the following system

$$
\begin{aligned}
x_{k+1} & =f\left(x_{k}, u_{k}, w_{k}\right), \quad k \geq 0, \\
z_{k} & =g\left(x_{k}\right), \quad k \geq 0 .
\end{aligned}
$$

For the measurement feedback uniform $l^{\infty}$-bounded (ULIB) synthesis problem, we consider the following system

$$
\begin{aligned}
x_{k+1} & =f\left(x_{k}, u_{k}, w_{k}\right), \quad k \geq 0, \\
y_{k} & =h\left(x_{k}, w_{k}\right), \quad k \geq 0, \\
z_{k} & =g\left(x_{k}\right), \quad k \geq 0 .
\end{aligned}
$$

Here $x_{k} \in \mathbf{R}^{n}, u_{k} \in \mathbf{U} \subseteq \mathbf{R}^{m}, w_{k} \in \mathbf{W} \subseteq \mathbf{R}^{s}$ are the state, control input, and disturbance input, respectively. $z_{k} \in \mathbf{R}$ is the performance output quantity. In (16), $y_{k} \in \mathbf{R}^{p}$ is the measured output.

We still use the same notation $\mathcal{C}_{s f}, \mathcal{C}_{m f}$ and $\phi\left(k, x_{0}, u, w_{[0, k-1]}\right)$ as those in the $\mathrm{SF} \star$ and $\mathrm{MF} \star$ problems (though the systems considered here are a bit different).

State Feedback ULIB (SFULIB) Problem: Consider system (15) and let $B_{0} \subseteq \mathbf{R}^{n}$ and $\lambda \in \mathbf{R}$ be given. Find, if possible, an admissible state feedback controller $K \in \mathcal{C}_{s f}$ such that the trajectories of the closed-loop system consisting of the plant (15) and the controller $K(\cdot)$ satisfy

$$
g\left(\phi\left(k, x_{0}, u, w_{[0, k-1]}\right)\right) \leq \lambda, \quad \forall x_{0} \in B_{0}, \forall w_{[0, k-1]} \in \mathcal{W}_{[0, k-1]}, \forall k \geq 0 .
$$

When there exists such a controller, we say that the SFULIB Problem is solvable for system (15).

Measurement Feedback ULIB (MFULIB) Problem: Consider system (16) and let $B_{0} \subseteq \mathbf{R}^{n}$ and $\lambda \in \mathbf{R}$ be given. Find, if possible, an admissible measurement feedback 
controller $K \in \mathcal{C}_{m f}$ such that the trajectories of the closed-loop system consisting of the plant (16) and the controller $K(\cdot)$ satisfy (17). When there exists such a controller, we say that the MFULIB Problem is solvable for system (16).

Remark 4.5 Solutions to the SFULIB Problem and MFULIB Problem were obtained in [12]. Note the similarity between the bounds in (12) and (17) that are respectively used to define the $\mathrm{SF} \star \mathrm{MF} \star$ and ULIB problems. The main difference is that the bound in (12) depends directly on $\phi\left(k, x_{0}, u, w_{[0, k-1]}\right), \rho^{\star}\left(x_{0}, k\right), \psi_{k}^{\star}\left(w_{[0, k-1]}\right)$ and $\varphi_{k}^{\star}\left(\phi_{[0, k-1]}\right)$ whereas the bound in (17) depends only on $\phi\left(k, x_{0}, u, w_{[0, k-1]}\right)$. However, we will show in the next section that $\rho^{\star}\left(x_{0}, k\right), \psi_{k}^{\star}\left(w_{[0, k-1]}\right)$ and $\varphi_{k}^{\star}\left(x_{[0, k-1]}\right)$ for any property given in Table 1 can be generated as solutions of auxiliary difference equations that are appropriately initialized and, moreover, we can solve the $\mathrm{SF} \star$ Problem for the system (10) and the MF $\star$ Problem for the system (13) by solving appropriate ULIB problems for augmented auxiliary systems that is appropriately initialized. This "problem transformation" is discussed in the next section.

\section{Problem Transformations}

In this section we show how the $\mathrm{SF} \star$ Problem for the system (10) and the MF $\star$ Problem for the system (13) can be converted into appropriate ULIB problems for auxiliary augmented systems.

\subsection{State Feedback Case}

In this section, we will use Tables $1,2,3,4$ to introduce an auxiliary system that will be useful in solving SF $\star$ Problem. Let $\star \in\{$ ISS, iISS, iIiSS, IOS, IOSS, $\delta$ ISS $\}$ be given. Let $n^{\star}$ and $G^{\star}: \mathbf{R}^{n^{\star}} \rightarrow \mathbf{R}$ come from Table 1 , where $n^{\star}$ denotes the dimension of the auxiliary system. Let functions $f_{\rho}^{\star}: \mathbf{R}_{+} \rightarrow \mathbf{R}_{+}, \hat{w}^{\star}: \mathbf{R}^{n} \times \mathbf{R}^{m} \times \mathbf{R}^{n} \rightarrow \mathbf{R}_{+}, \hat{f}_{\psi}^{\star}: \mathbf{R}_{+} \times \mathbf{R}_{+} \rightarrow$ $\mathbf{R}_{+}, f_{\varphi}^{\star}: \mathbf{R}_{+} \times \mathbf{R}^{n} \rightarrow \mathbf{R}_{+}$and $\zeta_{0}^{\star}, \hat{\eta}_{0}^{\star}, \theta_{0}^{\star} \in \mathbf{R}$ come from Tables $2,3,4$. We define the following auxiliary system

$$
\begin{aligned}
\hat{\xi}_{k+1}^{\star} & =\hat{f}^{\star}\left(\hat{\xi}_{k}^{\star}, u_{k}, w_{k}\right), \quad k \geq 0 \\
z_{k} & =G^{\star}\left(\hat{\xi}_{k}^{\star}\right), \quad k \geq 0
\end{aligned}
$$

where

$$
\hat{\xi}^{\star}:=\left(\begin{array}{c}
x \\
\zeta^{\star} \\
\hat{\eta}^{\star} \\
\theta^{\star}
\end{array}\right), \hat{f}^{\star}\left(\hat{\xi}^{\star}, u, w\right):=\left(\begin{array}{c}
f(x, u, w) \\
f_{\rho}^{\star}\left(\zeta^{\star}\right) \\
\hat{f}_{\psi}^{\star}\left(\hat{\eta}^{\star}, \hat{w}^{\star}(x, u, f(x, u, w))\right) \\
f_{\varphi}^{\star}\left(\theta^{\star}, x\right)
\end{array}\right)
$$

We also let:

$$
\hat{B}_{0}^{\star}:=\left\{\left(\begin{array}{c}
x_{0} \\
\zeta_{0}^{\star} \\
\hat{\eta}_{0}^{\star} \\
\theta_{0}^{\star}
\end{array}\right): x_{0} \in B_{0}\right\}, \quad \lambda:=0 .
$$




\begin{tabular}{||c|c|c|c|c||}
\hline \hline $\begin{array}{c}\text { Property } \\
\star\end{array}$ & $\rho^{\star}\left(x_{0}, k\right)=\zeta_{k}^{\star}$ & $\zeta_{0}^{\star}$ & dynamics of $\zeta_{k}^{\star}$ & $f_{\rho}^{\star}\left(\zeta^{\star}\right)$ \\
\hline \hline $\begin{array}{c}\text { ISS } \\
\text { IISS } \\
\text { IOS } \\
\text { IOSS }\end{array}$ & $\alpha_{2}\left(\left|x_{0}\right|\right) e^{-k}$ & $\alpha_{2}\left(\left|x_{0}\right|\right)$ & $\zeta_{k+1}^{\star}=e^{-1} \zeta_{k}^{\star}$ & $e^{-1} \zeta^{\star}$ \\
\hline $\operatorname{IIiSS}$ & $\gamma_{3}\left(\left|x_{0}\right|\right)$ & $\gamma_{3}\left(\left|x_{0}\right|\right)$ & $\zeta_{k+1}^{\star}=\zeta_{k}^{\star}$ & $\zeta^{\star}$ \\
\hline$\delta \operatorname{ISS}$ & $\alpha_{2}\left(\left|x_{0}^{1}-x_{0}^{2}\right|\right) e^{-k}$ & $\alpha_{2}\left(\left|x_{0}^{1}-x_{0}^{2}\right|\right)$ & $\zeta_{k+1}^{\star}=e^{-1} \zeta_{k}^{\star}$ & $e^{-1} \zeta^{\star}$ \\
\hline \hline
\end{tabular}

Table 2: Summary of the variable $\zeta_{k}^{\star}$ and the function $f_{\rho}^{\star}$ in equations $(18),(19),(44),(45)$

Remark 5.1 Here we use $n^{\star}$ to denote the dimension of the auxiliary system (18)(19). We can see that this dimension depend on the property $\star$. For example, $n^{\star}=$ $n+3$ when $\star \in\{$ iIiSS,IOSS $\} \quad\left(\right.$ where $\left.\hat{\xi}^{\star}=\left(x, \zeta^{\star}, \hat{\eta}^{\star}, \theta^{\star}\right)\right) ; n^{\star}=n+2$ when $\star \epsilon$ $\{I S S, i I S S, I O S, \delta I S S\}$ (when $\hat{\xi}^{\star}=\left(x, \zeta^{\star}, \hat{\eta}^{\star}\right)$, the variable $\theta^{\star}$ is not needed), see Tables 1 and 4 . When considering some different properties other than those listed in Table 1 , the dimension $n^{\star}$ may also be different. For example, when $\star=G A S$ (Global Asymptotic Stability, which can be obtained from ISS with $w \equiv 0), n^{\star}=n+1$. In this paper, we were not concentrating on the GAS property but the tools can be easily used to address it.

The main result of this subsection is stated below which shows a relationship of the $\mathrm{SF} \star$ Problem for system (10) and the SFULIB Problem for auxiliary system (18)-(19) with $\hat{B}_{0}^{\star}$ and $\lambda$ defined in (20). Since the system (18)-(19) is higher dimensional than (10), we find it convenient to introduce different notation for sets of admissible state feedback controllers. The sets of admissible state feedback controllers for (18)-(19) and (10) are respectively denoted as $\overline{\mathcal{C}}_{s f}^{\star}$ and $\mathcal{C}_{s f}$. i.e.

$$
\overline{\mathcal{C}}_{s f}^{\star}:=\left\{\bar{K}: \overline{\mathcal{X}}_{[0, \infty)}^{\star} \rightarrow \mathcal{U}_{[0, \infty)}, \bar{K} \text { is causal }\right\},
$$

where $\overline{\mathcal{X}}_{[0, \infty)}^{\star}$ is defined similarly as in (3) with $\bar{X}^{\star}=\mathbf{R}^{n^{\star}}$.

Theorem 5.2 Let $B_{0} \subseteq \mathbf{R}^{n}, \mathbf{W} \subseteq \mathbf{R}^{s}$ and $\mathbf{U} \subseteq \mathbf{R}^{m}$ be given. Let $\star \in\{I S S$, iISS, iIiSS, IOS, IOSS, $\delta I S S\}, \gamma_{i} \in \mathcal{K}, i=1,2, \ldots, 5, \alpha_{1}, \alpha_{2} \in \mathcal{K}_{\infty}$ be given and define $n^{\star}, G^{\star}, f_{\rho}^{\star}, \hat{w}^{\star}, \hat{f}_{\psi}^{\star}, f_{\varphi}^{\star}, \zeta_{0}^{\star}, \hat{\eta}_{0}^{\star}$ and $\theta_{0}^{\star}$ as generated by Tables 1,2,3,4. Let $X=\mathbf{R}^{n}, \bar{X}^{\star}=\mathbf{R}^{n^{\star}}$ and define the sets of admissible controllers $\mathcal{C}_{s f}, \overline{\mathcal{C}}_{\text {sf }}^{\star}$ by (11),(21). Then the following statements are equivalent: 


\begin{tabular}{||c|c|c|c|c||}
\hline \hline $\begin{array}{c}\text { Property } \\
\star\end{array}$ & $\hat{w}^{\star}\left(x_{0}, u_{0}, x_{1}\right)$ & $\hat{\eta}_{0}^{\star}$ & dynamics of $\hat{\eta}_{k}^{\star}$ & $\hat{f}_{\psi}^{\star}\left(\hat{\eta}^{\star}, \hat{w}^{\star}\right)$ \\
\hline \hline $\begin{array}{c}\text { ISS } \\
\text { IOS } \\
\text { IOSS }\end{array}$ & $\min _{w \in \mathbf{W}}\left\{|w|: f\left(x_{0}, u_{0}, w\right)=x_{1}\right\}$ & 0 & $\hat{\eta}_{k+1}^{\star}=\max \left\{\hat{\eta}_{k}^{\star}, \hat{w}^{\star}\left(x_{k}, u_{k}, x_{k+1}\right)\right\}$ & $\max \left\{\hat{\eta}^{\star}, \hat{w}^{\star}\right\}$ \\
\hline $\operatorname{IISS}$ & $\min _{w \in \mathbf{W}}\left\{|w|: f\left(x_{0}, u_{0}, w\right)=x_{1}\right\}$ & 0 & $\hat{\eta}_{k+1}^{\star}=\hat{\eta}_{k}^{\star}+\gamma_{2}\left(\hat{w}^{\star}\left(x_{k}, u_{k}, x_{k+1}\right)\right)$ & $\hat{\eta}^{\star}+\gamma_{2}\left(\hat{w}^{\star}\right)$ \\
\hline $\operatorname{IIiSS}$ & $\min _{w \in \mathbf{W}}\left\{|w|: f\left(x_{0}, u_{0}, w\right)=x_{1}\right\}$ & 0 & $\hat{\eta}_{k+1}^{\star}=\hat{\eta}_{k}^{\star}+\gamma_{5}\left(\hat{w}^{\star}\left(x_{k}, u_{k}, x_{k+1}\right)\right)$ & $\hat{\eta}^{\star}+\gamma_{5}\left(\hat{w}^{\star}\right)$ \\
\hline$\delta$ ISS & $\min _{w \in \mathbf{W}}\left\{\left|w^{1}-w^{2}\right|: f\left(x_{0}, u_{0}, w\right)=x_{1}\right\}$ & 0 & $\hat{\eta}_{k+1}^{\star}=\max \left\{\hat{\eta}_{k}^{\star}, \hat{w}^{\star}\left(x_{k}, u_{k}, x_{k+1}\right)\right\}$ & $\max \left\{\hat{\eta}^{\star}, \hat{w}^{\star}\right\}$ \\
\hline \hline
\end{tabular}

Table 3: Summary of the variable $\hat{\eta}_{k}^{\star}$ and functions $\hat{w}^{\star}$ and $\hat{f}_{\psi}^{\star}$ in equations (18),(19)

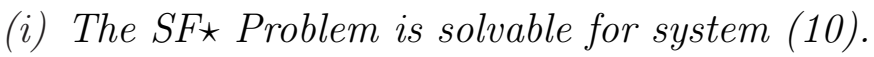

(ii) The SFULIB Problem is solvable for system (18)-(19) with $\hat{B}_{0}^{\star}$ and $\lambda$ defined in (20).

Moreover, if controller $K \in \mathcal{C}_{s f}$ of the form

$$
u_{k}=K\left(x_{[0, k]}\right)
$$

solves the $S F \star$ Problem for system (10), then the controller $\bar{K} \in \overline{\mathcal{C}}_{\text {sf }}^{\star}$ defined by

$$
u_{k}=\bar{K}\left(\hat{\xi}_{[0, k]}^{\star}\right)=\bar{K}\left(x_{[0, k]}, \zeta_{[0, k]}^{\star}, \hat{\eta}_{[0, k]}^{\star}, \theta_{[0, k]}^{\star}\right):=K\left(x_{[0, k]}\right)
$$

solves the SFULIB Problem for system (18)-(19) with $\hat{B}_{0}^{\star}$ and $\lambda$ defined in (20). Conversely, if controller $\bar{K} \in \overline{\mathcal{C}}_{\text {sf }}^{\star}$ of the form

$$
u_{k}=\bar{K}\left(\hat{\xi}_{[0, k]}^{\star}\right)=\bar{K}\left(x_{[0, k]}, \zeta_{[0, k]}^{\star}, \hat{\eta}_{[0, k]}^{\star}, \theta_{[0, k]}^{\star}\right)
$$

solves the SFULIB Problem for the system (18)-(19) with $\hat{B}_{0}^{\star}$ and $\lambda$ defined in (20), then the following controller $K \in \mathcal{C}_{s f}$

$$
\left\{\begin{aligned}
\zeta_{k+1}^{\star} & =f_{\rho}^{\star}\left(\zeta_{k}^{\star}\right), \\
\hat{\eta}_{k+1}^{\star} & =\hat{f}_{\psi}^{\star}\left(\hat{\eta}_{k}^{\star}, \hat{w}^{\star}\left(x_{k}, u_{k}, x_{k+1}\right)\right), \\
\theta_{k+1}^{\star} & =f_{\varphi}^{\star}\left(\theta_{k}^{\star}, x_{k}\right), \\
u_{k} & =K\left(x_{[0, k]}, \zeta_{[0, k]}^{\star}, \hat{\eta}_{[0, k]}^{\star}, \theta_{[0, k]}^{\star}\right)
\end{aligned}\right.
$$

with initialization $\zeta_{0}^{\star}, \hat{\eta}_{0}^{\star}, \theta_{0}^{\star}$, solves the $S F \star$ Problem for system (10). 


\begin{tabular}{||c|c|c|c|c||}
\hline \hline $\begin{array}{c}\text { Property } \\
\star\end{array}$ & $\varphi_{k}^{\star}\left(\phi_{[0, k-1]}\right)=\theta_{k}^{\star}$ & $\theta_{0}^{\star}$ & dynamics of $\theta_{k}^{\star}$ & $f_{\varphi}^{\star}\left(\theta^{\star}, x\right)$ \\
\hline \hline $\begin{array}{c}\text { ISS } \\
\text { IISS } \\
\text { IOS } \\
\delta \text { ISS }\end{array}$ & --- & --- & --- & --- \\
\hline iIiSS & $\sum_{i=0}^{k-1} \gamma_{2}\left(\left|x_{i}\right|\right)$ & 0 & $\theta_{k+1}^{\star}=\theta_{k}^{\star}+\gamma_{2}\left(\left|x_{k}\right|\right)$ & $\theta^{\star}+\gamma_{2}(|x|)$ \\
\hline IOSS & $\left\|H(x)_{[0, k-1]}\right\|_{\infty}$ & 0 & $\theta_{k+1}^{\star}=\max \left\{\theta_{k}^{\star},\left|H\left(x_{k}\right)\right|\right\}$ & $\max \left\{\theta^{\star},|H(x)|\right\}$ \\
\hline \hline
\end{tabular}

Table 4: Summary of the variable $\theta_{k}^{\star}$ and the function $f_{\varphi}^{\star}$ in equations $(18),(19),(44),(45)$

The structure of the dynamic state feedback controller (25) for the case $\star=I S S$ is shown in Figure 1.

Proof. The SF $\star$ Problem for system (10) is to find a controller $K \in \mathcal{C}_{s f}$ such that the trajectory of the closed-loop system consisting of (10) and $K$ satisfies

$$
G^{\star}\left(x_{k}, \rho^{\star}\left(x_{0}, k\right), \psi_{k}^{\star}\left(w_{[0, k-1]}\right), \varphi_{k}^{\star}\left(x_{[0, k-1]}\right)\right) \leq 0,
$$

for all $x_{0} \in B_{0}, w_{[0, k-1]} \in \mathcal{W}_{[0, k-1]}, k \geq 0$.

We first prove that the inequality (26) is equivalent to

$$
G^{\star}\left(x_{k}, \rho^{\star}\left(x_{0}, k\right), \hat{\psi}_{k}^{\star}\left(x_{0}, w_{[0, k-1]}\right), \varphi_{k}^{\star}\left(x_{[0, k-1]}\right)\right) \leq 0,
$$

for all $x_{0} \in B_{0}, w_{[0, k-1]} \in \mathcal{W}_{[0, k-1]}, k \geq 0$, where $\hat{\psi}_{k}^{\star}\left(x_{0}, w_{[0, k-1]}\right):=\inf _{\tilde{w}_{[0, k-1]}}\left\{\psi_{k}^{\star}\left(\tilde{w}_{[0, k-1]}\right): \phi\left(i, x_{0}, u, \tilde{w}_{[0, k-1]}\right)=\phi\left(i, x_{0}, u, w_{[0, k-1]}\right), i=1, \cdots, k\right\}$.

i.e. $\hat{\psi}_{k}^{\star}\left(x_{0}, w_{[0, k-1]}\right)$ is the minimal possible $\psi_{k}^{\star}\left(\tilde{w}_{[0, k-1]}\right)$ where the disturbances $\tilde{w}_{[0, k-1]}$ and $w_{[0, k-1]}$ (with the same initial state $x_{0}$ ) result in the same state trajectory.

In fact, for the $G^{\star}$ in Table 1 , since $\gamma_{1}$ and $\gamma_{4}$ are class $\mathcal{K}$ functions, $G^{\star}$ is monotone in $\psi$, i.e.

$$
G^{\star}\left(x, \rho, \psi_{2}, \varphi\right) \leq G^{\star}\left(x, \rho, \psi_{1}, \varphi\right), \quad \forall x \in \mathbf{R}^{n}, \rho \geq 0, \phi \geq 0, \psi_{2} \geq \psi_{1} \geq 0 .
$$

If (27) holds, then from

$$
\hat{\psi}_{k}^{\star}\left(x_{0}, w_{[0, k-1]}\right) \leq \psi_{k}^{\star}\left(w_{[0, k-1]}\right)
$$




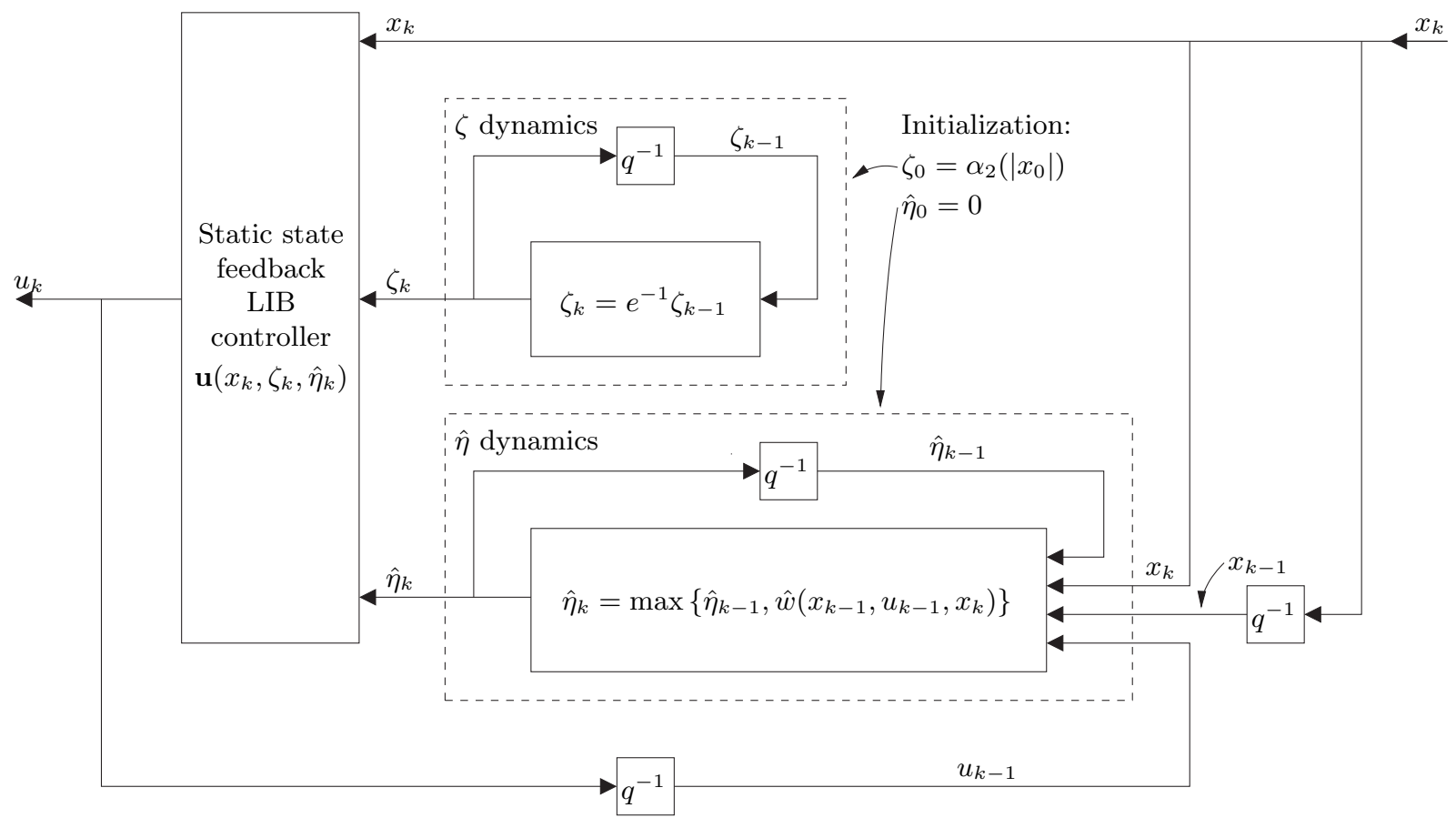

Figure 1: The dynamic state feedback controller (25) when $\star=I S S$ (see also (43) and (67)), where $q^{-1}$ denotes the unit step delay

we have (26). On the other hand, if (26) holds (for any $w_{[0, k-1]}$ ), then,

$$
\begin{aligned}
& G^{\star}\left(x_{k}, \rho^{\star}\left(x_{0}, k\right), \hat{\psi}_{k}^{\star}\left(x_{0}, w_{[0, k-1]}\right), \varphi_{k}^{\star}\left(x_{[0, k-1]}\right)\right) \\
= & \sup _{\tilde{w}_{[0, k-1]}}\left\{G^{\star}\left(x_{k}, \rho^{\star}\left(x_{0}, k\right), \psi_{k}^{\star}\left(\tilde{w}_{[0, k-1]}\right), \varphi_{k}^{\star}\left(x_{[0, k-1]}\right)\right)\right. \\
& \left.: \phi\left(i, x_{0}, u, \tilde{w}_{[0, k-1]}\right)=\phi\left(i, x_{0}, u, w_{[0, k-1]}\right), i=1, \cdots, k\right\} \\
\leq & 0 .
\end{aligned}
$$

Hence, (27) holds and this completes the proof of equivalence of (26) and (27).

Furthermore, notice that we can also write

$$
\hat{\psi}_{k}^{\star}\left(x_{0}, w_{[0, k-1]}\right)=\bar{\psi}_{k}^{\star}\left(x_{[0, k]}\right):=\inf _{\tilde{w}_{[0, k-1]}}\left\{\psi_{k}^{\star}\left(\tilde{w}_{[0, k-1]}\right): \phi\left(i, x_{0}, u, \tilde{w}_{[0, i-1]}\right)=x_{i}, i=1, \cdots, k\right\},
$$

where $x_{i}=\phi\left(i, x_{0}, u, w_{[0, i-1]}\right), i=1, \cdots, k$ is the state sequence which is available for feedback. Hence, (27) is further equivalent to

$$
G^{\star}\left(x_{k}, \rho^{\star}\left(x_{0}, k\right), \bar{\psi}_{k}^{\star}\left(x_{[0, k]}\right), \varphi_{k}^{\star}\left(x_{[0, k-1]}\right)\right) \leq 0,
$$

for all $x_{0} \in B_{0}, w_{[0, k-1]} \in \mathcal{W}_{[0, k-1]}, k \geq 0$.

Notice that the left hand side of (30) is a function of the state trajectory $x_{[0, k]}$. As long as the state sequence $x_{[0, k]}$ is available for feedback, the four items $x_{k}, \rho^{\star}\left(x_{0}, k\right)$, $\bar{\psi}_{k}^{\star}\left(x_{[0, k]}\right), \varphi_{k}^{\star}\left(x_{[0, k-1]}\right)$ can all be computed and, thus, are available for feedback. This makes it possible to transfer the SF $\star$ Problem for system (10) into a SFULIB problem for the auxiliary system. 
In fact, we can use three difference equations to generate the terms $\rho^{\star}\left(x_{0}, k\right), \hat{\psi}_{k}^{\star}\left(x_{0}, w_{[0, k-1]}\right)$ and $\varphi_{k}^{\star}\left(x_{[0, k-1]}\right)$ in the inequality $(27)$, respectively. The details are stated next. Some important functions used in this procedure are summarized in Tables 2,3,4.

For each property $\star \in\{I S S$, iISS, iIiSS, IOS, IOSS, $\delta I S S\}$, we define a new variable $\zeta_{k}^{\star}$, the initial value $\zeta_{0}^{\star}$ is given in the 3 th column of Table 2 , the dynamics of $\zeta_{k}^{\star}$ is given in the 4 th column of Table 2 . Similarly, define a new variable $\hat{\eta}_{k}^{\star}$, the initial state $\hat{\eta}_{0}^{\star}$ is given in the 3 th column of Table 3 , the dynamics of $\hat{\eta}_{k}^{\star}$ is given in the 4th column of Table 3 where the function $\hat{w}^{\star}$ is given in the 2 th column of Table 3 . Define a new variable $\theta_{k}^{\star}$, the initial state $\theta_{0}^{\star}$ is given in the 3 th column of Table 4 , the dynamics of $\theta_{k}^{\star}$ is given in the 4 th column of Table 4 .

By defining the new variables in this way, we have

$$
\zeta_{k}^{\star}=\rho^{\star}\left(x_{0}, k\right), \hat{\eta}_{k}^{\star}=\hat{\psi}_{k}^{\star}\left(x_{0}, w_{[0, k-1]}\right), \theta_{k}^{\star}=\varphi_{k}^{\star}\left(x_{[0, k-1]}\right) .
$$

Hence, the requirement (26) (or (27)) is equivalent to

$$
G^{\star}\left(\hat{\xi}_{k}^{\star}\right) \leq \lambda, \quad \forall \hat{\xi}_{0}^{\star} \in \hat{B}_{0}^{\star}, \forall w_{[0, k-1]} \in \mathcal{W}_{[0, k-1]}, k \geq 0 .
$$

where $\hat{\xi}^{\star}$ is defined in (19), $\hat{B}_{0}^{\star}$ and $\lambda$ are given in (20). This is actually the requirement in SFULIB Problem for system (18)-(19). (Notice that the dynamics of $\hat{\eta}_{k}^{\star}$ is $\hat{\eta}_{k+1}^{\star}=$ $\hat{f}_{\psi}^{\star}\left(\hat{\eta}_{k}^{\star}, \hat{w}^{\star}\left(x_{k}, u_{k}, x_{k+1}\right)\right)=\hat{f}_{\psi}^{\star}\left(\hat{\eta}_{k}^{\star}, \hat{w}^{\star}\left(x_{k}, u_{k}, f\left(x_{k}, u_{k}, w_{k}\right)\right)\right)$.)

Now it is not hard to prove the theorem. If controller $K \in \mathcal{C}_{s f}$ of the form (22) solves the SF $\star$ Problem for system (10), then the closed-loop system combining (10) with the control input sequence obtained by (22) satisfies (26). Thus the closed-loop system combining (18)-(19) with the same control input sequence satisfies (31). Notice that this control input sequence can also be obtained by the map $\bar{K} \in \overline{\mathcal{C}}_{s f}^{\star}$ defined by (23). Hence $\bar{K}$ solves the SFULIB Problem for system (18)-(19) with $\hat{B}_{0}^{\star}$ and $\lambda$ defined in (20).

Conversely, if controller $\bar{K} \in \overline{\mathcal{C}}_{s f}^{\star}$ of the form (24) solves the SFULIB Problem for the system (18)-(19) with $\hat{B}_{0}^{\star}$ and $\lambda$ defined in (20), then the closed-loop system combining (18)-(19) with the control input sequence obtained by (24) satisfies (31). Hence the closedloop system combining (10) with the same control input sequence satisfies (26). Notice that this control input sequence can also be obtained by the map $K \in \mathcal{C}_{s f}$ defined by (25) with initialization $\zeta_{0}^{\star}, \hat{\eta}_{0}^{\star}, \theta_{0}^{\star}$. Hence $K \in \mathcal{C}_{s f}$ solves the $\mathrm{SF} \star$ Problem for system (10).

To illustrate how Tables 1,2,3,4 are used in Theorem 5.2, consider the case when $\star=$ ISS. For simplicity, we will omit the supscript "ISS" in the expressions, e.g. use $G$ instead of $G^{I S S}$, etc. Notice that the variable $\theta$ is not needed in this case (see Table 4 ).

From Table 1, row 1 column 5, we have

$$
G(x, \zeta, \eta)=|x|-\alpha_{1}(\zeta)-\gamma_{1}(\eta)
$$

From Tables 2 and 3, row 1, we have

$$
\begin{gathered}
f_{\rho}(\zeta)=e^{-1} \zeta, \quad \hat{f}_{\psi}(\eta, w)=\max \{\eta,|w|\}, \\
\zeta_{0}=\alpha_{2}\left(\left|x_{0}\right|\right), \quad \hat{\eta}_{0}=0,
\end{gathered}
$$




$$
\hat{w}\left(x_{0}, u_{0}, x_{1}\right)=\min _{w \in \mathbf{W}}\left\{|w|: f\left(x_{0}, u_{0}, w\right)=x_{1}\right\} .
$$

Hence (19) takes the form

$$
\hat{\xi}=\left(\begin{array}{c}
x \\
\zeta \\
\hat{\eta}
\end{array}\right), \quad \hat{f}(\hat{\xi}, u, w)=\left(\begin{array}{c}
f(x, u, w) \\
e^{-1} \zeta \\
\max \left\{\hat{\eta}, \min _{\tilde{w} \in \mathbf{W}}\{|\tilde{w}|: f(x, u, \tilde{w})=f(x, u, w)\}\right\}
\end{array}\right) .
$$

So the auxiliary system (18) becomes

$$
\left\{\begin{aligned}
x_{k+1} & =f\left(x_{k}, u_{k}, w_{k}\right), \\
\zeta_{k+1} & =e^{-1} \zeta_{k} \\
\hat{\eta}_{k+1} & =\max \left\{\hat{\eta}_{k}, \min _{w \in \mathbf{W}}\left\{|w|: f\left(x_{k}, u_{k}, w\right)=f\left(x_{k}, u_{k}, w_{k}\right)\right\}\right\} \\
z_{k} & =\left|x_{k}\right|-\alpha_{1}\left(\zeta_{k}\right)-\gamma_{1}\left(\hat{\eta}_{k}\right) .
\end{aligned}\right.
$$

Also, (20) becomes in this case

$$
\hat{B}_{0}=\left\{\left(\begin{array}{c}
x_{0} \\
\alpha_{2}\left(\left|x_{0}\right|\right) \\
0
\end{array}\right): x_{0} \in B_{0}\right\}, \quad \lambda=0
$$

The set of admissible state feedback controllers for the auxiliary system (37) is

$$
\overline{\mathcal{C}}_{s f}:=\left\{\bar{K}: \overline{\mathcal{X}}_{[0, \infty)} \rightarrow \mathcal{U}_{[0, \infty)}, \bar{K} \text { is causal }\right\},
$$

where $\overline{\mathcal{X}}_{[0, \infty)}$ is defined similarly as in (3) with $\bar{X}=\mathbf{R}^{n+2}$.

Corollary 5.3 (ISS Case) Let $B_{0} \subseteq \mathbf{R}^{n}, \mathbf{W} \subseteq \mathbf{R}^{s}, \mathbf{U} \subseteq \mathbf{R}^{m}, \gamma_{1} \in \mathcal{K}$ and $\alpha_{1}, \alpha_{2} \in \mathcal{K}_{\infty}$ be given. Let $X=\mathbf{R}^{n}, \bar{X}=\mathbf{R}^{n+2}$ and define the sets of admissible controllers $\mathcal{C}_{s f}, \overline{\mathcal{C}}_{s f}$ by (11),(39). Then, the following statements are equivalent:

(i) The SFISS Problem is solvable for system (10).

(ii) The SFULIB Problem is solvable for system (37) with $\hat{B}_{0}$ and $\lambda$ defined in (38).

Moreover, if controller $K \in \mathcal{C}_{\text {sf }}$ of the form

$$
u_{k}=K\left(x_{[0, k]}\right)
$$

solves the SFISS Problem for system (10), then the controller $\bar{K} \in \overline{\mathcal{C}}_{\text {sf }}$ defined by

$$
u_{k}=\bar{K}\left(x_{[0, k]}, \zeta_{[0, k]}, \hat{\eta}_{[0, k]}\right):=K\left(x_{[0, k]}\right)
$$

solves the SFULIB Problem for system (37) with $\hat{B}_{0}^{\star}$ and $\lambda$ defined in (38). Conversely, if controller $\bar{K} \in \overline{\mathcal{C}}_{s f}$ of the form

$$
u_{k}=\bar{K}\left(x_{[0, k]}, \zeta_{[0, k]}, \hat{\eta}_{[0, k]}\right)
$$

solves the SFULIB Problem for the system (37) with $\hat{B}_{0}^{\star}$ and $\lambda$ defined in (38), then the following controller $K \in \mathcal{C}_{s f}$

$$
\left\{\begin{aligned}
\zeta_{k+1} & =e^{-1} \zeta_{k}, \\
\hat{\eta}_{k+1} & =\max \left\{\hat{\eta}_{k}, \min _{w \in \mathbf{W}}\left\{|w|: f\left(x_{k}, u_{k}, w\right)=x_{k+1}\right\}\right\} \\
u_{k} & =\bar{K}\left(x_{[0, k]}, \zeta_{[0, k]}, \hat{\eta}_{[0, k]}\right)
\end{aligned}\right.
$$

with initialization $\zeta_{0}=\alpha_{2}\left(\left|x_{0}\right|\right), \hat{\eta}_{0}=0$, solves the SFISS Problem for system (10). 


\begin{tabular}{||c|c|c|c|c||}
\hline \hline $\begin{array}{c}\text { Property } \\
\star\end{array}$ & $\psi_{k}^{\star}\left(w_{[0, k-1]}\right)=\eta_{k}^{\star}$ & $\eta_{0}^{\star}$ & dynamics of $\eta_{k}^{\star}$ & $f_{\psi}^{\star}\left(\eta^{\star}, w\right)$ \\
\hline \hline $\begin{array}{c}\text { ISS } \\
\text { IOS } \\
\text { OOSS }\end{array}$ & $\left\|w_{[0, k-1]}\right\|_{\infty}$ & 0 & $\eta_{k+1}^{\star}=\max \left\{\eta_{k}^{\star},\left|w_{k}\right|\right\}$ & $\max \left\{\eta^{\star},|w|\right\}$ \\
\hline $\operatorname{IISS}$ & $\sum_{i=0}^{k-1} \gamma_{2}\left(\left|w_{i}\right|\right)$ & 0 & $\eta_{k+1}^{\star}=\eta_{k}^{\star}+\gamma_{2}\left(\left|w_{k}\right|\right)$ & $\eta^{\star}+\gamma_{2}(|w|)$ \\
\hline $\operatorname{iIiSS}$ & $\sum_{i=0}^{k-1} \gamma_{5}\left(\left|w_{i}\right|\right)$ & 0 & $\eta_{k+1}^{\star}=\eta_{k}^{\star}+\gamma_{5}\left(\left|w_{k}\right|\right)$ & $\eta^{\star}+\gamma_{5}(|w|)$ \\
\hline$\delta \mathrm{ISS}$ & $\left\|\left(w^{1}-w^{2}\right)_{[0, k-1]}\right\|_{\infty}$ & 0 & $\eta_{k+1}^{\star}=\max \left\{\eta_{k}^{\star},\left|w_{k}^{1}-w_{k}^{2}\right|\right\}$ & $\max \left\{\eta^{\star},\left|w^{1}-w^{2}\right|\right\}$ \\
\hline \hline
\end{tabular}

Table 5: Summary of the variable $\eta_{k}^{\star}$ and the function $f_{\psi}^{\star}$ in equations $(44),(45)$

\subsection{Measurement Feedback Case}

In this section, we will use Tables $1,2,4,5$ to introduce an auxiliary system that will be useful in solving MF $\star$ Problem. Let $\star \in\{$ ISS, iISS, iIiSS, IOS, IOSS, $\delta$ ISS $\}$ be given. Let $n^{\star}$ and $G^{\star}: \mathbf{R}^{n^{\star}} \rightarrow \mathbf{R}$ come from Table 1, where $n^{\star}$ denotes the dimension of the auxiliary system. Let functions $f_{\rho}^{\star}: \mathbf{R} \rightarrow \mathbf{R}, f_{\psi}^{\star}: \mathbf{R} \times \mathbf{R}^{s} \rightarrow \mathbf{R}, f_{\varphi}^{\star}: \mathbf{R} \times \mathbf{R}^{n} \rightarrow \mathbf{R}$, and $\zeta_{0}^{\star}, \theta_{0}^{\star}, \eta_{0}^{\star} \in \mathbf{R}$ come from Tables $2,4,5$. We define the following auxiliary system

$$
\begin{aligned}
\xi_{k+1}^{\star} & =\tilde{f}^{\star}\left(\xi_{k}^{\star}, u_{k}, w_{k}\right), \quad k \geq 0 \\
z_{k} & =G^{\star}\left(\xi_{k}^{\star}\right), \quad k \geq 0 \\
y_{k} & =h\left(x_{k}, w_{k}\right), \quad k \geq 0
\end{aligned}
$$

where

$$
\xi^{\star}:=\left(\begin{array}{c}
x \\
\zeta^{\star} \\
\eta^{\star} \\
\theta^{\star}
\end{array}\right), \quad \tilde{f}^{\star}\left(\xi^{\star}, u, w\right):=\left(\begin{array}{c}
f(x, u, w) \\
f_{\rho}^{\star}\left(\zeta^{\star}\right) \\
f_{\psi}^{\star}\left(\eta^{\star}, w\right) \\
f_{\varphi}^{\star}\left(\theta^{\star}, x\right)
\end{array}\right)
$$

We also let:

$$
\tilde{B}_{0}^{\star}:=\left\{\left(\begin{array}{c}
x_{0} \\
\zeta_{0}^{\star} \\
\eta_{0}^{\star} \\
\theta_{0}^{\star}
\end{array}\right): x_{0} \in B_{0}\right\}, \quad \lambda:=0 .
$$

The following theorem shows a relationship of the MF $\star$ Problem for system (13) and the MFULIB Problem for auxiliary system (44)-(45) with $\tilde{B}_{0}^{\star}$ and $\lambda$ defined in (46). 
Theorem 5.4 Let $\mathbf{Y}=$ range $\{h\} \subseteq \mathbf{R}^{p}$ and $\mathbf{U} \subseteq \mathbf{R}^{m}$ be given and define the set of admissible controller $\mathcal{C}_{m f}$ as in (14). Let $B_{0} \subseteq \mathbf{R}^{n}, \mathbf{W} \subseteq \mathbf{R}^{s}, \star \in\{I S S$, iISS, iIiSS, IOS, IOSS, $\delta I S S\}, \gamma_{i} \in \mathcal{K}, i=1,2, \ldots, 5, \alpha_{1}, \alpha_{2} \in \mathcal{K}_{\infty}$ be given and define $n^{\star}, G^{\star}, f_{\rho}^{\star}, f_{\psi}^{\star}, f_{\varphi}^{\star}, \zeta_{0}^{\star}, \theta_{0}^{\star}$ and $\eta_{0}^{\star}$ as generated by Tables 1,2,4,5. Then, the following statements are equivalent:

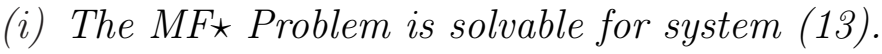

(ii) The MFULIB Problem is solvable for system (44)-(45) with $\tilde{B}_{0}^{\star}$ and $\lambda$ defined in (46).

Moreover, a controller $K \in \mathcal{C}_{m f}$ of the form

$$
u_{k}=K\left(y_{[0, k-1]}\right)
$$

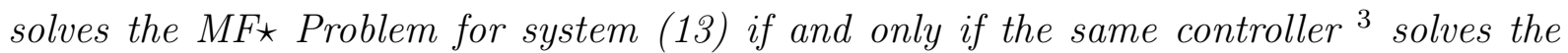
MFULIB Problem for the system (44)-(45) with $\tilde{B}_{0}^{\star}$ and $\lambda$ defined in (46).

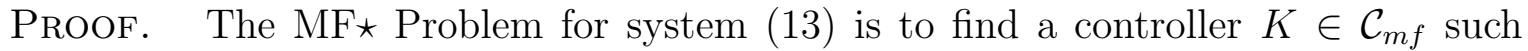
that the trajectory of the closed-loop system consisting of (13) and $K$ satisfies (26). Now we only need to introduce three new variables $\zeta_{k}^{\star}, \eta_{k}^{\star}, \theta_{k}^{\star} \in \mathbf{R}$ to characterize the terms $\rho^{\star}\left(x_{0}, k\right), \psi_{k}^{\star}\left(w_{[0, k-1]}\right)$ and $\varphi_{k}^{\star}\left(x_{[0, k-1]}\right)$ in the inequality $(26)$, respectively. This time we will make use of Tables $2,4,5$.

For each property $\star \in\{I S S$, iISS, iIiSS, IOS, IOSS, $\delta I S S\}$, we define the new variable $\zeta_{k}^{\star}=\rho^{\star}\left(x_{0}, k\right)$, the initial value $\zeta_{0}^{\star}$ and the dynamics of $\zeta_{k}^{\star}$ are given in the 3 th column and 4th column of Table 2 . Similarly, define the variable $\theta_{k}^{\star}=\varphi_{k}^{\star}\left(x_{[0, k-1]}\right)$, the initial state $\theta_{0}^{\star}$ and the dynamics of $\theta_{k}^{\star}$ are given in the 3th column and 4 th column of Table 4. Define the new variable $\eta_{k}^{\star}=\psi_{k}^{\star}\left(w_{[0, k-1]}\right)$, the initial state $\eta_{0}^{\star}$ and the dynamics of $\eta_{k}^{\star}$ are given in the 3 th column and 4 th column of Table 5 .

Now the inequality (26) is equivalent to

$$
G^{\star}\left(\xi_{k}^{\star}\right) \leq \lambda, \quad \forall \xi_{0}^{\star} \in \tilde{B}_{0}^{\star}, \forall w_{[0, k-1]} \in \mathcal{W}_{[0, k-1]}, k \geq 0 .
$$

where $\xi^{\star}$ is defined in (45), $\tilde{B}_{0}^{\star}$ and $\lambda$ are given in (46). This is the requirement in MFULIB Problem for system (44)-(45).

Notice that the system (13) and the system (44)-(45) have the same control input $u$ and the same measured output $y$, so the set of the admissible controllers for the MF $\star$ Problem for system (13) and the set of the admissible controllers for the MFULIB Problem for system (44)-(45) are both $\mathcal{C}_{m f}$. We can assert the theorem from the equivalence of (48) and (26).

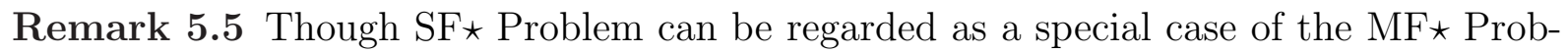
lem, we treat the two problems separately because we can obtain much better solution when the plant states are available for the controller. Especially, (1) for the problem

\footnotetext{
${ }^{3}$ Notice that the dimensions of the measurement outputs of system (13) and system (44)-(45) are the same, the dimensions of the control inputs of system (13) and system (44)-(45) are also the same. Here "the same controller" means the mapping from the measurement output to control input is the same.
} 
transformation, the auxiliary states used for the $\mathrm{SF} \star$ Problem is different from the aux-

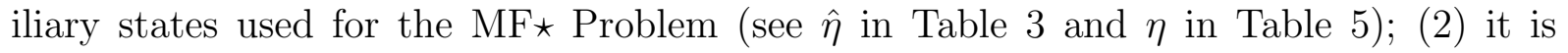
shown in Theorem 5.2 that the SF $\star$ Problem can be transferred into a SFULIB problem instead of a MFULIB problem; (3) as shown in [12], the controllers for SFULIB problem are state feedback controllers which are much easier to implement than the information state feedback controllers for MFULIB problem.

To illustrate how Tables 1,2,4,5 are used in Theorem 5.4, consider the case when $\star=$ ISS. As before, we will omit the supscript "ISS" in the expressions, e.g. use $G$ instead of $G^{I S S}$, etc.

From Table 1, row 1, we have

$$
G(x, \zeta, \eta)=|x|-\alpha_{1}(\zeta)-\gamma_{1}(\eta)
$$

By Tables 2 and 5, row 1, we have

$$
f_{\rho}(\zeta)=e^{-1} \zeta, \quad f_{\psi}(\eta, w)=\max \{\eta,|w|\}, \quad \zeta_{0}=\alpha_{2}\left(\left|x_{0}\right|\right), \quad \eta_{0}=0 .
$$

Hence (45) takes the form

$$
\xi=\left(\begin{array}{c}
x \\
\zeta \\
\eta
\end{array}\right), \quad \tilde{f}(\xi, u, w)=\left(\begin{array}{c}
f(x, u, w) \\
e^{-1} \zeta \\
\max \{\eta,|w|\}
\end{array}\right)
$$

So the auxiliary system (44)-(45) becomes

$$
\left\{\begin{aligned}
x_{k+1} & =f\left(x_{k}, u_{k}, w_{k}\right) \\
\zeta_{k+1} & =e^{-1} \zeta_{k} \\
\eta_{k+1} & =\max \left\{\eta_{k},\left|w_{k}\right|\right\} \\
z_{k} & =\left|x_{k}\right|-\alpha_{1}\left(\zeta_{k}\right)-\gamma_{1}\left(\eta_{k}\right) \\
y_{k} & =h\left(x_{k}, w_{k}\right)
\end{aligned}\right.
$$

Also, (46) becomes

$$
\tilde{B}_{0}=\left\{\left(\begin{array}{c}
x_{0} \\
\alpha_{2}\left(\left|x_{0}\right|\right) \\
0
\end{array}\right): x_{0} \in B_{0}\right\}, \quad \lambda=0
$$

Corollary 5.6 (ISS Case) Let $\mathbf{Y}=$ range $\{h\} \subseteq \mathbf{R}^{p}$ and $\mathbf{U} \subseteq \mathbf{R}^{m}$ be given and define the set of admissible controller $\mathcal{C}_{m f}$ as in (14). Let $B_{0} \subseteq \mathbf{R}^{n}, \mathbf{W} \subseteq \mathbf{R}^{s}, \gamma_{1} \in \mathcal{K}, \alpha_{1}, \alpha_{2} \in \mathcal{K}_{\infty}$ be given. Then, the following statements are equivalent:

(i) The MFISS Problem is solvable for system (13).

(ii) The MFULIB Problem is solvable for system (52) with $\tilde{B}_{0}$ and $\lambda$ defined in (53).

Moreover, a controller $K \in \mathcal{C}_{m f}$ of the form

$$
u_{k}=K\left(y_{[0, k-1]}\right)
$$

solves the MFISS Problem for system (13) if and only if the same controller $K$ solves the MFULIB Problem for the system (52) with $\tilde{B}_{0}$ and $\lambda$ defined in (53). 


\section{Dynamic Programming Results}

Using Theorems 5.2 and 5.4 and the results of ULIB problems [12], we have the following dynamic programming results for the $\mathrm{SF} \star$ and $\mathrm{MF} \star$ problems. The results in this section are direct consequences of Theorems 3.3, 3.5, 4.17, 4.19 in [12]. The dynamic programming inequalities provide a framework for controller design to achieve various ISS like properties in terms of Lyapunov-like storage functions (numerical methods may need to solve for them).

\subsection{State Feedback Case}

Theorem 6.1 (Necessity) Let $B_{0} \subseteq \mathbf{R}^{n}, \mathbf{W} \subseteq \mathbf{R}^{s}, \mathbf{U} \subseteq \mathbf{R}^{m}$ be given. Let $\star \in\{I S S$, $i I S S$, iIiSS, IOS, IOSS, $\delta I S S\}, \gamma_{i} \in \mathcal{K}, i=1,2, \ldots, 5, \alpha_{1}, \alpha_{2} \in \mathcal{K}_{\infty}$ be given and define $n^{\star}, G^{\star}, f_{\rho}^{\star}, \hat{w}^{\star}, \hat{f}_{\psi}^{\star}, f_{\varphi}^{\star}, \zeta_{0}^{\star}, \hat{\eta}_{0}^{\star}$ and $\theta_{0}^{\star}$ as generated by Tables 1,2,3,4. Let $\hat{f}^{\star}$ come from (19), let $\hat{B}_{0}^{\star}$ and $\lambda$ come from (20). Let $X=\mathbf{R}^{n}, \bar{X}^{\star}=\mathbf{R}^{n^{\star}}$ and define the sets of admissible controllers $\mathcal{C}_{s f}, \overline{\mathcal{C}}_{s f}^{\star}$ by (11),(21). If the $S F \star$ Problem for system (10) is solvable, then the value function $V_{a}^{\star}: \mathbf{R}^{n^{\star}} \rightarrow \overline{\mathbf{R}}$ defined by ${ }^{4}$

$$
V_{a}^{\star}\left(\hat{\xi}^{\star}\right):=\inf _{\bar{K} \in \overline{\mathcal{C}}_{s f}^{\star}} \sup _{k \geq 0} \sup _{w_{[0, k-1]} \in \mathcal{W}_{[0, k-1]}}\left\{G^{\star}\left(\hat{\xi}_{k}^{\star}\right): u_{k}=\bar{K}\left(\hat{\xi}_{[0, k]}^{\star}\right), \hat{\xi}_{0}^{\star}=\hat{\xi}^{\star}\right\}, \forall \hat{\xi}^{\star} \in \mathbf{R}^{n^{\star}}
$$

satisfies:

1. $\hat{B}_{0}^{\star} \subseteq \operatorname{dom} V_{a}^{\star}:=\left\{\hat{\xi}^{\star} \in \mathbf{R}^{n^{\star}}: V_{a}^{\star}\left(\hat{\xi}^{\star}\right)<+\infty\right\} ;$

2. $\sup _{\hat{\xi}^{\star} \in \hat{B}_{0}^{\star}} V_{a}^{\star}\left(\hat{\xi}^{\star}\right) \leq \lambda$; $\hat{\xi}^{\star} \in \hat{B}_{0}^{\star}$

3. the following dynamic programming equation (DPE) holds

$$
V_{a}^{\star}\left(\hat{\xi}^{\star}\right)=\max \left\{G^{\star}\left(\hat{\xi}^{\star}\right), \inf _{u \in \mathbf{U}} \sup _{w \in \mathbf{W}} V_{a}^{\star}\left(\hat{f}^{\star}\left(\hat{\xi}^{\star}, u, w\right)\right)\right\}, \forall \hat{\xi}^{\star} \in \operatorname{dom} V_{a}^{\star} .
$$

Proof. Suppose there exists a $K_{0} \in \mathcal{C}_{s f}$ solving the SF $\star$ Problem for system (10). Then from Theorem 5.2, there exists a $\bar{K}_{0} \in \mathcal{C}_{s f}^{\star}$ solving the SFULIB Problem for system (18)-(19) with $\hat{B}_{0}^{\star}$ and $\lambda$ defined in (20). By Theorem 3.3 in [12], the items 1 and 3 in Theorem 6.1 hold. By the definition of $V_{a}^{\star}$,

$$
V_{a}^{\star}\left(\hat{\xi}^{\star}\right) \leq \sup _{k \geq 0} \sup _{w_{[0, k-1]} \in \mathcal{W}_{[0, k-1]}}\left\{G^{\star}\left(\hat{\xi}_{k}^{\star}\right): u_{k}=\bar{K}_{0}\left(\hat{\xi}_{[0, k]}^{\star}\right), \hat{\xi}_{0}^{\star}=\hat{\xi}^{\star}\right\}, \forall \hat{\xi}^{\star} \in \mathbf{R}^{n^{\star}}
$$

Because $\bar{K}_{0}$ solves the SFULIB Problem for system (18)-(19) with $\hat{B}_{0}^{\star}$ and $\lambda$, we have

$$
\sup _{k \geq 0} \sup _{w_{[0, k-1]} \in \mathcal{W}_{[0, k-1]}}\left\{G^{\star}\left(\hat{\xi}_{k}^{\star}\right): u_{k}=\bar{K}_{0}\left(\hat{\xi}_{[0, k]}^{\star}\right), \hat{\xi}_{0}^{\star}=\hat{\xi}^{\star}\right\} \leq \lambda, \forall \hat{\xi}^{\star} \in \hat{B}_{0}^{\star} .
$$

Thus the item 2 in Theorem 6.1 holds.

${ }^{4} \overline{\mathcal{C}}_{s f}^{\star}$ is the set of admissible state feedback controller (21) for system (18)-(19), $\mathcal{W}_{[0, k-1]}$ is defined in (3), $\hat{\xi}_{k}^{\star}$ is the solution of system (18)-(19) with $u_{k}=\bar{K}\left(\hat{\xi}_{[0, k]}^{\star}\right)$ and $\hat{\xi}_{0}^{\star}=\hat{\xi}^{\star}$. 
Theorem 6.2 (Sufficiency) Let $B_{0} \subseteq \mathbf{R}^{n}, \mathbf{W} \subseteq \mathbf{R}^{s}$ and $\mathbf{U} \subseteq \mathbf{R}^{m}$ be given. Let $\star \in\{$ ISS, $i I S S$, iIiSS, IOS, IOSS, $\delta I S S\}, \gamma_{i} \in \mathcal{K}, i=1,2, \ldots, 5, \alpha_{1}, \alpha_{2} \in \mathcal{K}_{\infty}$ be given and define $n^{\star}, G^{\star}, f_{\rho}^{\star}, \hat{w}^{\star}, \hat{f}_{\psi}^{\star}, f_{\varphi}^{\star}, \zeta_{0}^{\star}, \hat{\eta}_{0}^{\star}$ and $\theta_{0}^{\star}$ as generated by Tables 1,2,3,4. Let $\hat{f}^{\star}$ come from (19), let $\hat{B}_{0}^{\star}$ and $\lambda$ come from (20). Let $X=\mathbf{R}^{n}, \bar{X}^{\star}=\mathbf{R}^{n^{\star}}$ and define the sets of admissible controllers $\mathcal{C}_{s f}, \overline{\mathcal{C}}_{s f}^{\star}$ by (11),(21). Suppose that there exist $S \subseteq \mathbf{R}^{n^{\star}}, V^{\star}: \mathbf{R}^{n^{\star}} \rightarrow \overline{\mathbf{R}}$ and $\mathbf{u}^{\star}: S \rightarrow \mathbf{U}$ such that the following conditions hold:

1. $\hat{B}_{0}^{\star} \subseteq S$;

2. $\sup V^{\star}\left(\hat{\xi}^{\star}\right) \leq \lambda$;

$\hat{\xi}^{\star} \in S$

3. the following dynamic programming inequality (DPI) holds

$$
V^{\star}\left(\hat{\xi}^{\star}\right) \geq \max \left\{G^{\star}\left(\hat{\xi}^{\star}\right), \inf _{u \in \mathbf{U}} \sup _{w \in \mathbf{W}} V^{\star}\left(\hat{f}^{\star}\left(\hat{\xi}^{\star}, u, w\right)\right)\right\}, \forall \hat{\xi}^{\star} \in S
$$

4. for all $\hat{\xi}^{\star} \in S$,

$$
\max \left\{G^{\star}\left(\hat{\xi}^{\star}\right), \sup _{w \in \mathbf{W}} V^{\star}\left(\hat{f}^{\star}\left(\hat{\xi}^{\star}, \mathbf{u}^{\star}\left(\hat{\xi}^{\star}\right), w\right)\right)\right\}=\max \left\{G^{\star}\left(\hat{\xi}^{\star}\right), \inf _{u \in \mathbf{U}} \sup _{w \in \mathbf{W}} V^{\star}\left(\hat{f}^{\star}\left(\hat{\xi}^{\star}, u, w\right)\right)\right\} ;
$$

5. the solution $\hat{\xi}_{k}^{\star}$ of

$$
\hat{\xi}_{k+1}^{\star}=\hat{f}^{\star}\left(\hat{\xi}_{k}^{\star}, \mathbf{u}^{\star}\left(\hat{\xi}_{k}^{\star}\right), w_{k}\right)
$$

satisfy

$$
\hat{\xi}_{k}^{\star} \in S
$$

for all $\hat{\xi}_{0}^{\star} \in S, k \geq 0$ and $w_{[0, k-1]} \in \mathcal{W}_{[0, k-1]}$.

Then, the following controller $K^{\star} \in \mathcal{C}_{\text {sf }}$ defined by

$$
\left\{\begin{aligned}
\zeta_{k+1}^{\star} & =f_{\rho}^{\star}\left(\zeta_{k}^{\star}\right), \\
\hat{\eta}_{k+1}^{\star} & =\hat{f}_{\psi}^{\star}\left(\hat{\eta}_{k}^{\star}, \hat{w}^{\star}\left(x_{k}, u_{k}, x_{k+1}\right)\right), \\
\theta_{k+1}^{\star} & =f_{\varphi}^{\star}\left(\theta_{k}^{\star}, x_{k}\right) \\
u_{k} & =\mathbf{u}^{\star}\left(\hat{\xi}_{k}^{\star}\right)=\mathbf{u}^{\star}\left(x_{k}, \zeta_{k}^{\star}, \hat{\eta}_{k}^{\star}, \theta_{k}^{\star}\right)
\end{aligned}\right.
$$

with initialization $\zeta_{0}^{\star}, \hat{\eta}_{0}^{\star}, \theta_{0}^{\star}$, solves the $S F \star$ Problem for system (10).

Proof. By Conditions 3,4,5 we have that the pair $\left(V^{\star}, S\right)$ is a "good solution" of the DPI (57) in the sense of Definition 3.4 in [12]. Denote

$$
\bar{K}\left(\hat{\xi}_{[0, k]}^{\star}\right):=\mathbf{u}^{\star}\left(\hat{\xi}_{k}^{\star}\right)
$$

then by Conditions 1,2 and Theorem 3.5 in [12], $\bar{K}$ solves the SFULIB Problem for system (18)-(19) with $\hat{B}_{0}^{\star}$ and $\lambda$ defined in (20). By Theorem 5.2, controller $K \in \mathcal{C}_{s f}$ defined by (61) with initialization $\zeta_{0}^{\star}, \hat{\eta}_{0}^{\star}, \theta_{0}^{\star}$, solves the $\mathrm{SF} \star$ Problem for system (10). 
Remark 6.3 There are gaps between the necessity result (Theorem 6.1) and the sufficiency result (Theorem 6.2). For example, (i) the value function $V_{a}^{\star}$ in Theorem 6.1 is the minimal value function while the value function $V^{\star}$ in Theorem 6.2 may not be the minimal; (ii) the value function $V_{a}^{\star}$ satisfies the DPE while the value function $V^{\star}$ is only required to satisfy the DPI; (iii) if a controller $K_{0}$ solves the the $\mathrm{SF} \star$ Problem for system (10), it may not be of the form (61) in Theorem 6.2. Due to these gaps between the necessity and sufficiency, Theorems 6.1 and 6.2 can not be combined into one theorem. Similarly, Theorems 6.6 and 6.7 in the next section can not be combined into one theorem.

Theorem 6.1 and Theorem 6.2 can be regarded as 6 different results. For example, when $\star=$ ISS, functions $G$ and $\hat{f}$ are given in (32) and (36), respectively. Also, $\hat{B}_{0}$ and $\lambda$ are given in (38). So we have the following corollary by Theorem 6.2.

Corollary 6.4 (ISS case, State Feedback, Sufficiency) Let $B_{0} \subseteq \mathbf{R}^{n}, \mathbf{W} \subseteq \mathbf{R}^{s}, \mathbf{U} \subseteq \mathbf{R}^{m}$, $\gamma_{1} \in \mathcal{K}$ and $\alpha_{1}, \alpha_{2} \in \mathcal{K}_{\infty}$ be given. Let $X=\mathbf{R}^{n}, \bar{X}=\mathbf{R}^{n+2}$ and define the sets of admissible controllers $\mathcal{C}_{s f}, \overline{\mathcal{C}}_{s f}$ by (11),(39). Suppose that there exist $S \subseteq \mathbf{R}^{n+2}, V$ : $\mathbf{R}^{n+2} \rightarrow \overline{\mathbf{R}}$ and $\mathbf{u}: S \rightarrow \mathbf{U}$ such that the following conditions hold:

1. $\left\{\left(\begin{array}{c}x_{0} \\ \alpha_{2}\left(\left|x_{0}\right|\right) \\ 0\end{array}\right): x_{0} \in B_{0}\right\} \subseteq S$;

2. $\sup _{(x, \zeta, \hat{\eta}) \in S} V(x, \zeta, \hat{\eta}) \leq 0$;

3. the following DPI holds

$$
\begin{array}{r}
V(x, \zeta, \hat{\eta}) \geq \max \left\{|x|-\alpha_{1}(\zeta)-\gamma_{1}(\hat{\eta}) \inf _{u \in \mathbf{U}} \sup _{w \in \mathbf{W}} V\left(f(x, u, w), e^{-1} \zeta, f_{\eta}(\hat{\eta}, x, u, w)\right)\right\} \\
\forall(x, \zeta, \hat{\eta}) \in S ;
\end{array}
$$

where

$$
f_{\eta}(\hat{\eta}, x, u, w)=\max \left\{\hat{\eta}, \min _{\tilde{w} \in \mathbf{W}}\{|\tilde{w}|: f(x, u, \tilde{w})=f(x, u, w)\}\right\}
$$

4. for all $(x, \zeta, \hat{\eta}) \in S$,

$$
\begin{aligned}
& \max \left\{|x|-\alpha_{1}(\zeta)-\gamma_{1}(\hat{\eta}), \sup _{w \in \mathbf{W}} V\left(f(x, \mathbf{u}(x, \zeta, \hat{\eta}), w), e^{-1} \zeta, f_{\eta}(\hat{\eta}, x, \mathbf{u}(x, \zeta, \hat{\eta}), w)\right)\right\} \\
= & \max \left\{|x|-\alpha_{1}(\zeta)-\gamma_{1}(\hat{\eta}), \inf _{u \in \mathbf{U}} \sup _{w \in \mathbf{W}} V\left(f(x, u, w), e^{-1} \zeta, f_{\eta}(\hat{\eta}, x, u, w)\right)\right\} ;
\end{aligned}
$$

where $f_{\eta}(\hat{\eta}, x, u, w)$ is given in (63).

5. the solution $\left(x_{k}, \zeta_{k}, \hat{\eta}_{k}\right)$ of

$$
\left\{\begin{aligned}
x_{k+1} & =f\left(x_{k}, \mathbf{u}\left(x_{k}, \zeta_{k}, \hat{\eta}_{k}\right), w_{k}\right) \\
\zeta_{k+1} & =e^{-1} \zeta_{k} \\
\hat{\eta}_{k+1} & =\max \left\{\hat{\eta}_{k}, \min _{w \in \mathbf{W}}\left\{|w|: f\left(x_{k}, \mathbf{u}\left(x_{k}, \zeta_{k}, \hat{\eta}_{k}\right), w\right)=x_{k+1}\right\}\right\}
\end{aligned}\right.
$$


satisfy

$$
\left(x_{k}, \zeta_{k}, \hat{\eta}_{k}\right) \in S
$$

for all $\left(x_{0}, \zeta_{0}, \hat{\eta}_{0}\right) \in S, k \geq 0$ and $w_{[0, k-1]} \in \mathcal{W}_{[0, k-1]}$.

Then, the following controller $K \in \mathcal{C}_{\text {sf }}$ defined by

$$
\left\{\begin{aligned}
\zeta_{k+1} & =e^{-1} \zeta_{k}, \\
\hat{\eta}_{k+1} & =\max \left\{\hat{\eta}_{k}, \min _{w \in \mathbf{W}}\left\{|w|: f\left(x_{k}, u_{k}, w\right)=x_{k+1}\right\}\right\}, \\
u_{k} & =\mathbf{u}\left(x_{k}, \zeta_{k}, \hat{\eta}_{k}\right)
\end{aligned}\right.
$$

with initialization $\zeta_{0}=\alpha_{2}\left(\left|x_{0}\right|\right), \hat{\eta}_{0}=0$, solves the SFISS Problem for system (10).

\subsection{Measurement Feedback Case}

Let $n^{\star}$ be given. We use $2^{\mathbf{R}^{n^{\star}}}$ to denote the set of all subsets of $\mathbf{R}^{n^{\star}}$. For given functions $G^{\star}: \mathbf{R}^{n^{\star}} \rightarrow \mathbf{R}, f_{\rho}^{\star}: \mathbf{R} \rightarrow \mathbf{R}, f_{\psi}^{\star}: \mathbf{R} \times \mathbf{R}^{s} \rightarrow \mathbf{R}, f_{\varphi}^{\star}: \mathbf{R} \times \mathbf{R}^{n} \rightarrow \mathbf{R}$, we define $\hat{G}^{\star}: 2^{\mathbf{R}^{n^{\star}}} \rightarrow \mathbf{R}$ by

$$
\hat{G}^{\star}(X):=\sup _{\xi^{\star} \in X} G^{\star}\left(\xi^{\star}\right), \quad \forall X \subseteq \mathbf{R}^{n^{\star}}
$$

and $F^{\star}: 2^{\mathbf{R}^{n^{\star}}} \times \mathbf{R}^{m} \times \mathbf{R}^{p} \rightarrow 2^{\mathbf{R}^{n^{\star}}}$ by

$$
\begin{aligned}
F^{\star}(X, u, y):= & \left\{(x, \zeta, \eta, \theta): \exists w \in \mathbf{W}, \exists\left(x^{\prime}, \zeta^{\prime}, \eta^{\prime}, \theta^{\prime}\right) \in X, \text { such that } h\left(x^{\prime}, w\right)=y,\right. \\
& \left.f\left(x^{\prime}, u, w\right)=x, f_{\rho}^{\star}\left(\zeta^{\prime}\right)=\zeta, f_{\psi}^{\star}\left(\eta^{\prime}, w\right)=\eta, f_{\varphi}^{\star}\left(\theta^{\prime}, x^{\prime}\right)=\theta\right\} .
\end{aligned}
$$

The set-valued observer is defined as

$$
X_{i+1}=F\left(X_{i}, u_{i}, y_{i}\right), \quad X_{0} \subseteq \mathbf{R}^{n^{\star}} .
$$

Remark 6.5 The solution of set-valued observer are sets which are estimations of the states of system (44)-(45). In fact, for $X_{0} \subseteq \mathbf{R}^{n^{\star}}, j \geq 1, u_{[0, j-1]} \in \mathcal{U}_{[0, j-1]}, y_{[0, j-1]} \in \mathcal{Y}_{[0, j-1]}$,

$$
\begin{gathered}
X_{j}=\left\{(x, \zeta, \eta, \theta): \exists w_{[0, j-1]} \in \mathcal{W}_{[0, j-1]}, \exists\left(x_{0}, \zeta_{0}, \eta_{0}, \theta_{0}\right) \in X_{0},\right. \text { such that } \\
x_{j}=x, \zeta_{j}=\zeta, \eta_{j}=\eta, \theta_{j}=\theta, h\left(x_{i}, w_{i}\right)=y_{i}, 0 \leq i \leq j-1, \\
\text { where } x_{i+1}=f\left(x_{i}, u_{i}, w_{i}\right), \zeta_{i+1}=f_{\rho}^{\star}\left(\zeta_{i}\right), \eta_{i+1}=f_{\psi}^{\star}\left(\eta_{i}, w_{i}\right) \\
\left.\theta_{i+1}=f_{\varphi}^{\star}\left(\theta_{i}, x_{i}\right), 0 \leq i \leq j-1\right\} .
\end{gathered}
$$

Using Theorem 5.4 and Theorems 4.17 and 4.19 in [12], we can obtain the dynamic programming results for the MF $\star$ Problem.

Theorem 6.6 (Necessity) Let $\mathbf{Y}=$ range $\{h\} \subseteq \mathbf{R}^{p}$ and $B_{0} \subseteq \mathbf{R}^{n}, \mathbf{W} \subseteq \mathbf{R}^{s}, \mathbf{U} \subseteq \mathbf{R}^{m}$ be given and define the set of admissible controller $\mathcal{C}_{m f}$ as in (14). Let $\star \in\{I S S$, iISS, iI $S S$, IOS $, I O S S, \delta I S S\}, \gamma_{i} \in \mathcal{K}, i=1,2, \ldots, 5, \alpha_{1}, \alpha_{2} \in \mathcal{K}_{\infty}$ be given and define $n^{\star}, G^{\star}, f_{\rho}^{\star}, f_{\psi}^{\star}$, $f_{\varphi}^{\star}, \zeta_{0}^{\star}, \theta_{0}^{\star}$ and $\eta_{0}^{\star}$ as generated by Tables 1,2,4,5. Let $\tilde{f}^{\star}$ come from (45), let $\tilde{B}_{0}^{\star}$ and $\lambda$ come 


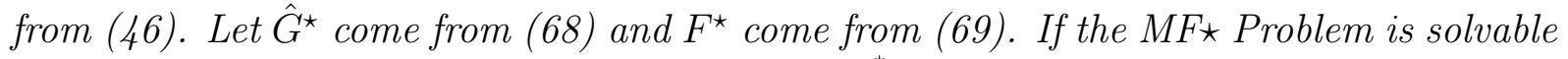
for system (13), then the value function $W_{a}^{\star}: 2^{\mathbf{R}^{n^{\star}}} \rightarrow \tilde{\mathbf{R}}$ defined by ${ }^{5}$

$$
W_{a}^{\star}(X):=\inf _{K \in \mathcal{C}_{m f}} \sup _{k \geq 0} \sup _{y_{[0, k-1]} \in \mathcal{Y}_{[0, k-1]}}\left\{\hat{G}^{\star}\left(X_{k}\right): X_{0}=X, u_{k}=K\left(y_{[0, k-1]}\right)\right\}
$$

satisfies

1. $\tilde{B}_{0}^{\star} \in \operatorname{dom} W_{a}^{\star}:=\left\{X \in 2^{\mathbf{R}^{n^{\star}}}:-\infty<W_{a}^{\star}(X)<+\infty\right\}$;

2. $W_{a}^{\star}\left(\tilde{B}_{0}^{\star}\right) \leq \lambda$;

3. the following dynamic programming equation (DPE) holds

$$
W_{a}^{\star}(X)=\max \left\{\hat{G}^{\star}(X), \inf _{u \in \mathbf{U}} \sup _{y \in \mathbf{Y}} W_{a}^{\star}\left(F^{\star}(X, u, y)\right)\right\}, \quad \forall X \in \operatorname{dom} W_{a}^{\star} .
$$

Proof. Suppose there exists a $K_{0} \in \mathcal{C}_{m f}$ solving the MF $\star$ Problem for system (13). Then from Theorem 5.4, $K_{0}$ solving the MFULIB Problem for system (44)-(45) with $\tilde{B}_{0}^{\star}$ and $\lambda$ defined in (46). The item 2 in Theorem 6.6 holds by the definition of $W_{a}^{\star}$,

$$
W_{a}^{\star}\left(\tilde{B}_{0}^{\star}\right) \leq \sup _{k \geq 0} \sup _{y_{[0, k-1]} \in \mathcal{Y}_{[0, k-1]}}\left\{\hat{G}^{\star}\left(X_{k}\right): X_{0}=\tilde{B}_{0}^{\star}, u_{k}=K_{0}\left(y_{[0, k-1]}\right)\right\} \leq \lambda .
$$

By Theorem 4.17 in [12], the items 1 and 3 in Theorem 6.6 hold.

Theorem 6.7 (Sufficiency) Let $\mathbf{Y}=$ range $\{h\} \subseteq \mathbf{R}^{p}$ and $B_{0} \subseteq \mathbf{R}^{n}, \mathbf{W} \subseteq \mathbf{R}^{s}, \mathbf{U} \subseteq \mathbf{R}^{m}$ be given and define the set of admissible controller $\mathcal{C}_{m f}$ as in (14). Let $\star \in\{I S S$, iISS, iIiSS, $I O S, I O S S, \delta I S S\}, \gamma_{i} \in \mathcal{K}, i=1,2, \ldots, 5, \alpha_{1}, \alpha_{2} \in \mathcal{K}_{\infty}$ be given and define $n^{\star}, G^{\star}, f_{\rho}^{\star}, f_{\psi}^{\star}$, $f_{\varphi}^{\star}, \zeta_{0}^{\star}, \theta_{0}^{\star}$ and $\eta_{0}^{\star}$ as generated by Tables 1,2,4,5. Let $\tilde{f}^{\star}$ come from (45), let $\tilde{B}_{0}^{\star}$ and $\lambda$ come from (46). Let $\hat{G}^{\star}$ come from (68) and $F^{\star}$ come from (69). Suppose there exist $\hat{S} \subseteq 2^{\mathbf{R}^{n^{\star}}}, W^{\star}: 2^{\mathbf{R}^{n^{\star}}} \rightarrow \tilde{\mathbf{R}}, \mathbf{u}^{\star}: \hat{S} \rightarrow \mathbf{U}$, and $X_{0} \in \hat{S}$ such that the following conditions hold:

1. $\tilde{B}_{0}^{\star} \subseteq X_{0}$;

2. $W^{\star}\left(X_{0}\right) \leq \lambda$;

3. the following DPI holds

$$
W^{\star}(X) \geq \max \left\{\hat{G}^{\star}(X), \inf _{u \in \mathbf{U}} \sup _{y \in \mathbf{Y}} W^{\star}\left(F^{\star}(X, u, y)\right)\right\}, \quad \forall X \in \hat{S}
$$

4. for all $X \in \hat{S}$,

$$
\max \left\{\hat{G}^{\star}(X), \sup _{y \in \mathbf{Y}} W^{\star}\left(F^{\star}\left(X, \mathbf{u}^{\star}(X), y\right)\right)\right\}=\max \left\{\hat{G}^{\star}(X), \inf _{u \in \mathbf{U}} \sup _{y \in \mathbf{Y}} W^{\star}(F(X, u, y))\right\} ;
$$

\footnotetext{
${ }^{5} \mathcal{C}_{m f}$ is the set of admissible measurement feedback controller (14) for system (44)-(45), $\mathcal{Y}_{[0, k-1]}$ is defined similarly as in (3), $X_{k}$ is the solution of (70) with $u_{k}=K\left(y_{[0, k-1]}\right)$ and $X_{0}=X$.
} 
5. the solution of

$$
X_{k+1}=F\left(X_{k}, \mathbf{u}^{\star}\left(X_{k}\right), y_{k}\right)
$$

with initial condition $X_{0} \in \hat{S}$ satisfies

$$
X_{k} \in \hat{S}
$$

for all $k \geq 0$ and $y_{[0, k-1]} \in \mathcal{Y}_{[0, k-1]}$.

Then the controller defined by

$$
u_{k}=\mathbf{u}^{\star}\left(X_{k}\right)
$$

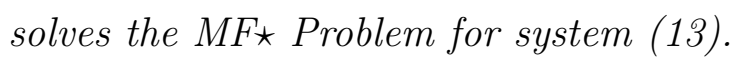

Proof. By Conditions $3,4,5$ we have that the pair $\left(W^{\star}, \hat{S}\right)$ is a "good solution" of the DPI (74) in the sense of Definition 4.18 in [12]. By Conditions 1,2 and Theorem 4.19 in [12], controller $K$ defined by (78) solves the MFULIB Problem for system (44)-(45) with $\tilde{B}_{0}^{\star}$ and $\lambda$ defined in (46). By Theorem 5.4, the same $K$ solves the MF $\star$ Problem for system (13).

Similarly, both Theorem 6.6 and Theorem 6.7 can be regarded as 6 different results. For example, when $\star=$ ISS, we can obtain the corresponding corollaries for the ISS case. The details are omitted here due to space limitations.

\section{Example}

Consider system

$$
x_{k+1}=x_{k}^{3}+x_{k}^{2} u_{k}+\sin \left(x_{k}\right) \cos \left(u_{k}\right)+\frac{2+\sin \left(x_{k}\right)}{1+u_{k}^{2}} w_{k} .
$$

We consider the SFISS problem with

$$
\alpha_{1}(s)=\alpha_{2}(s)=\gamma_{1}(s)=s .
$$

We use Corollary 6.4 to find a solution to the problem. For this example, we set

$$
B_{0}=[-1,1], \quad \mathbf{U}=[-1,1], \quad \mathbf{W}=[-1,1], \quad S=[-1,1] \times[0,1] \times[0,1] .
$$

Approximating Markov Chain method [15] is applied to solve the DPE obtained by changing the " $\geq$ " into "=" in the DPI (62). Notice that for this example, the function $f_{\eta}(\hat{\eta}, x, u, w)$ in (63) is simply

$$
f_{\eta}(\hat{\eta}, x, u, w)=\max \{\hat{\eta},|w|\} .
$$

Using the discretized space $\mathbf{U}, \mathbf{W}, S$ with grids of 40, we obtain an approximation for the value function $V(x, \zeta, \hat{\eta})$ and the optimal controller $\mathbf{u}(x, \zeta, \hat{\eta})$. For example, $V(x, \zeta, \hat{\eta})$ and $\mathbf{u}(x, \zeta, \hat{\eta})$ for $\hat{\eta}=0, \hat{\eta}=0.5, \hat{\eta}=1$ obtained in this way are illustrated in Figures 2.

A simulation of the state $x_{k}$ in the closed-loop system is illustrated in Figure 3 (we choose $x_{0}=0.8$ and a random disturbance sequence $w_{[0,20]}$ satisfying $\left.\left\|w_{[0,20]}\right\|_{\infty} \leq 0.2\right)$, which demonstrates consistency with the ISS inequality

$$
\left|x_{k}\right| \leq\left|x_{0}\right| e^{-k}+\left\|w_{[0, k-1]}\right\|_{\infty} .
$$




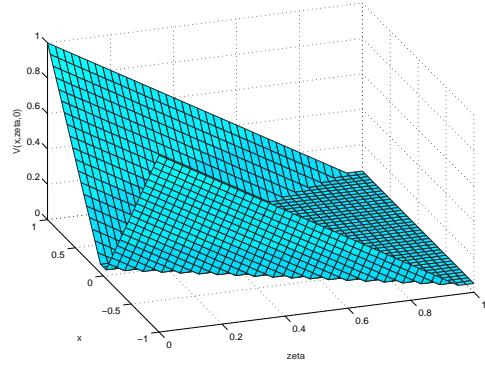

(a) $V(x, \zeta, 0)$

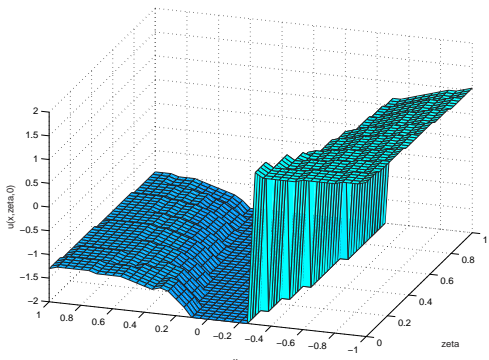

(d) $\mathbf{u}(x, \zeta, 0)$

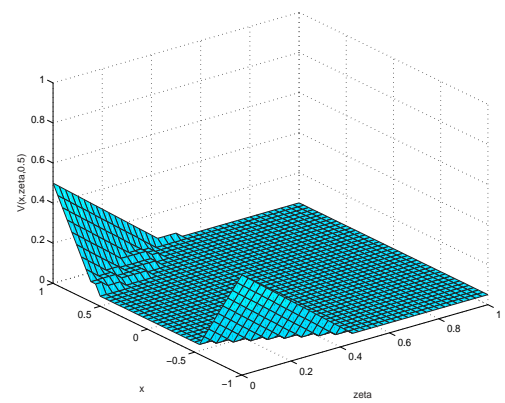

(b) $V(x, \zeta, 0.5)$

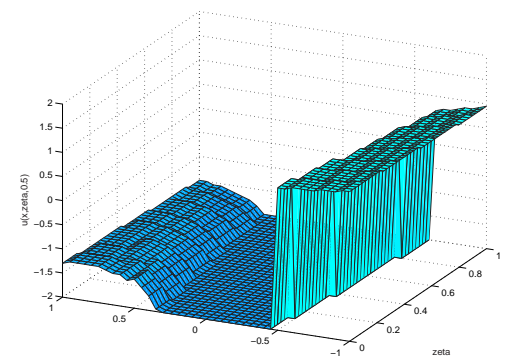

(e) $\mathbf{u}(x, \zeta, 0.5)$

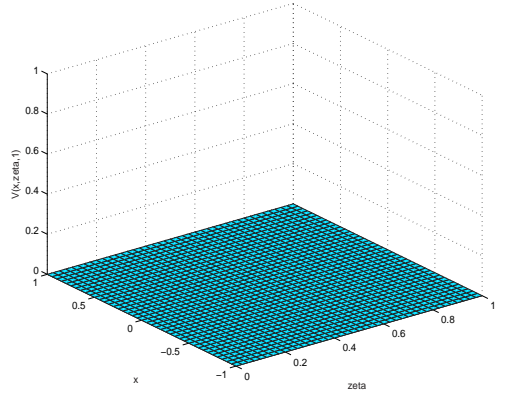

(c) $V(x, \zeta, 1)$

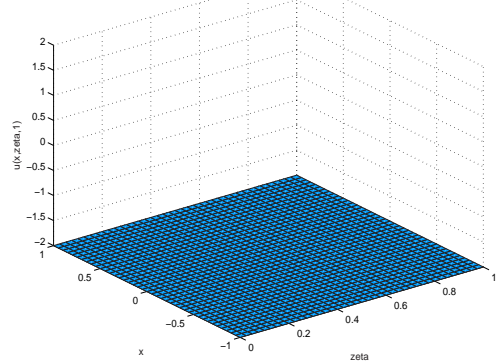

(f) $\mathbf{u}(x, \zeta, 1)$

Figure 2: Value function $V(x, \zeta, \hat{\eta})$ and State feedback controller $\mathbf{u}(x, \zeta, \hat{\eta})$
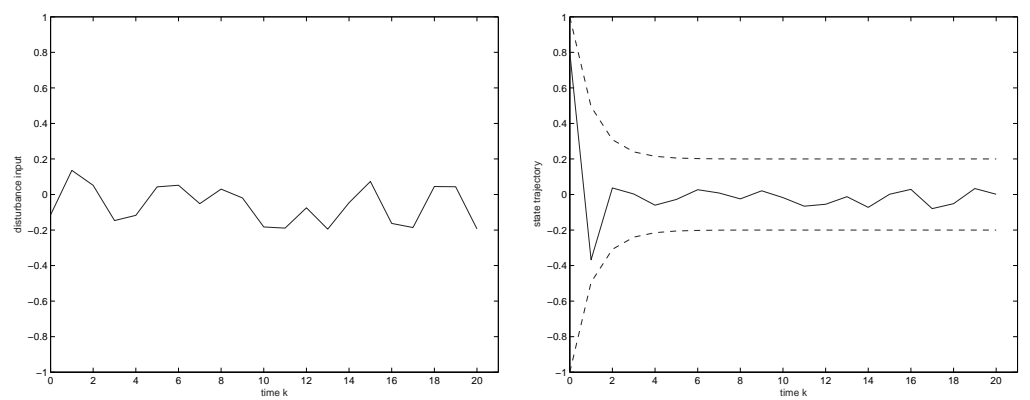

(a) disturbance input trajectory (b) state trajectory $x_{k}\left(x_{0}=0.8\right)$ $\left(\left\|w_{[0,20]}\right\|_{\infty} \leq 0.2\right)$

Figure 3: A trajectory of the closed-loop system 


\section{Further Remarks}

\subsection{Robustness to the initialization error of controller}

One feature of our method is initializing the states of the dynamic controller at certain values. Now we show that our design is actually robust to the small disturbances on these initialization. We illustrate this for the state feedback ISS case. The other cases can be dealt with similarly.

Suppose the conditions in Corollary 6.4 hold. With the dynamic state feedback controller (67), the overall closed-loop system is given by

$$
\left\{\begin{aligned}
x_{k+1} & =f\left(x_{k}, u_{k}, w_{k}\right) \\
\zeta_{k+1} & =e^{-1} \zeta_{k}, \\
\hat{\eta}_{k+1} & =\max \left\{\hat{\eta}_{k}, \min _{w \in \mathbf{W}}\left\{|w|: f\left(x_{k}, u_{k}, w\right)=x_{k+1}\right\}\right\} \\
u_{k} & =\mathbf{u}^{*}\left(x_{k}, \zeta_{k}, \hat{\eta}_{k}\right)
\end{aligned}\right.
$$

where $x_{0} \in B_{0}, \zeta_{0}=\alpha_{2}\left(\left|x_{0}\right|\right), \hat{\eta}_{0}=0$ and $x_{k} \in \mathbf{R}^{n}, k \geq 0$ are available.

Suppose there are disturbances on the initial state of the controller, the true initial states of the controller is given by

$$
\zeta_{0}=\alpha_{2}\left(\left|x_{0}\right|\right)+\delta \zeta, \quad \hat{\eta}_{0}=0+\delta \eta
$$

Since

$$
0 \leq \min _{w \in \mathbf{W}}\left\{|w|: f\left(x_{k}, u_{k}, w\right)=x_{k+1}\right\} \leq\left|w_{k}\right|
$$

we have

$$
\begin{aligned}
& \left|\hat{\eta}_{k}\right| \leq \max \left\{|\delta \eta|,\left|w_{0}\right|, \cdots,\left|w_{k-1}\right|\right\}=\max \left\{|\delta \eta|,\left\|w_{[0, k-1]}\right\|_{\infty}\right\} \\
& \left|\zeta_{k}\right|=e^{-k}\left|\zeta_{0}\right| \leq\left(\alpha_{2}\left(\left|x_{0}\right|\right)+|\delta \zeta|\right) e^{-k}
\end{aligned}
$$

Suppose $\left(x_{k}, \zeta_{k}, \hat{\eta}_{k}\right) \in S, \forall k \geq 0$, then from Conditions 2 and 3 in Corollary 6.4,

$$
\left|x_{k}\right|-\alpha_{1}\left(\zeta_{k}\right)-\gamma_{1}\left(\hat{\eta}_{k}\right) \leq V\left(x_{k}, \zeta_{k}, \hat{\eta}_{k}\right) \leq 0 .
$$

Hence we have

$$
\left|x_{k}\right| \leq \alpha_{1}\left(\zeta_{k}\right)+\gamma_{1}\left(\hat{\eta}_{k}\right) \leq \alpha_{1}\left(\left(\alpha_{2}\left(\left|x_{0}\right|\right)+|\delta \zeta|\right) e^{-k}\right)+\gamma_{1}\left(\max \left\{|\delta \eta|,\left\|w_{[0, k-1]}\right\|_{\infty}\right\}\right)
$$

Inequalities (86) and (87) show that when $|\delta \zeta|,|\delta \eta|$ are small, the changes on the upper bounds of $\left|\hat{\eta}_{k}\right|,\left|\zeta_{k}\right|,\left|x_{k}\right|$ are also small. Thus our design posses robustness with respect to controller initialization error to some extent.

\subsection{To achieve closed-loop ISS-like property}

The synthesis problems considered in this paper require only that a desired bound is achieved on the solutions of the plant states. In this section, we provide a technique that can be used in order to achieve the ISS-like property for the closed-loop systems involving 
the controller states. We illustrate this for the state feedback ISS case. The other cases can be dealt with similarly.

The idea is that we design our controller such that the plant state achieve a stronger property similar to the input-to-state dynamical stability property [9], then we are able to prove that the closed-loop system is actually ISS in the usual sense.

For the state feedback ISS case, we change the dynamics of $\zeta$ and $\hat{\eta}$ in (37) and consider the system

$$
\left\{\begin{aligned}
x_{k+1} & =f\left(x_{k}, u_{k}, w_{k}\right), \quad k \geq 0 \\
\zeta_{1} & =e^{-1}\left(\left|\zeta_{0}\right|+\alpha_{2}\left(\left|x_{0}\right|\right)\right) \\
\zeta_{k+1} & =e^{-1} \zeta_{k}, \quad k \geq 1 \\
\hat{\eta}_{k+1} & =\max \left\{a\left|\hat{\eta}_{k}\right|, \min _{w \in \mathbf{W}}\left\{|w|: f\left(x_{k}, u_{k}, w\right)=x_{k+1}\right\}\right\}, \quad k \geq 0
\end{aligned}\right.
$$

where $0<a<1$ and the initial conditions are $x_{0} \in B_{0}, \zeta_{0}, \hat{\eta}_{0} \in \mathbf{R}$. If we require

$$
G\left(x_{k}, \zeta_{k}, \hat{\eta}_{k}\right)=\left|x_{k}\right|-\alpha_{1}\left(\zeta_{k}\right)-\gamma_{1}\left(\hat{\eta}_{k}\right) \leq 0, \quad k \geq 1,
$$

for arbitrary $x_{0} \in B_{0}, \zeta_{0}, \hat{\eta}_{0} \in \mathbf{R}$, then, we can achieve closed-loop ISS property.

In fact, since

$$
0 \leq \min _{w \in \mathbf{W}}\left\{|w|: f\left(x_{k}, u_{k}, w\right)=f\left(x_{k}, u_{k}, w_{k}\right)\right\} \leq\left|w_{k}\right|
$$

we have

$$
\left|\hat{\eta}_{k+1}\right| \leq \max \left\{a\left|\hat{\eta}_{k}\right|,\left|w_{k}\right|\right\}, \quad k \geq 0
$$

and furthermore,

$$
\hat{\eta}_{k} \leq \max \left\{a^{k}\left|\hat{\eta}_{0}\right|,\left\|w_{[0, k-1]}\right\|_{\infty}\right\} \leq a^{k}\left|\hat{\eta}_{0}\right|+\left\|w_{[0, k-1]}\right\|_{\infty}, \quad k \geq 0 .
$$

Since we also have

$$
\zeta_{k} \leq e^{-k}\left(\left|\zeta_{0}\right|+\alpha_{2}\left(\left|x_{0}\right|\right)\right), \quad k \geq 0
$$

by (89) we get

$$
\begin{aligned}
\left|x_{k}\right| & \leq \alpha_{1}\left(\zeta_{k}\right)+\gamma_{1}\left(\hat{\eta}_{k}\right) \leq \alpha_{1}\left(e^{-k}\left(\left|\zeta_{0}\right|+\alpha_{2}\left(\left|x_{0}\right|\right)\right)+\gamma_{1}\left(\max \left\{a^{k}\left|\hat{\eta}_{0}\right|,\left\|w_{[0, k-1]}\right\|_{\infty}\right\}\right)\right. \\
& \leq \alpha_{1}\left(e^{-k}\left(\left|\zeta_{0}\right|+\alpha_{2}\left(\left|x_{0}\right|\right)\right)+\gamma_{1}\left(a^{k}\left|\hat{\eta}_{0}\right|\right)+\gamma_{1}\left(\left\|w_{[0, k-1]}\right\|_{\infty}\right\}\right), \quad k \geq 1 .
\end{aligned}
$$

Together with (91) and (92), we can get the closed-loop ISS inequality ( $a^{k} \rightarrow 0$ because $0<a<1)$.

Now the crucial part is to achieve the requirement (89). We can achieve this by applying our results on SFISS Problem twice as follows.

We choose the first control

$$
u_{0}=\mathbf{u}\left(x_{0}, \alpha_{2}\left(\left|x_{0}\right|\right), 0\right)
$$

where $\mathbf{u}(x, \zeta, \hat{\eta})$ is obtained by Corollary 6.4. Then for any $w_{0} \in \mathbf{W}, x_{1}=f\left(x_{0}, u_{0}, w_{0}\right)$ satisfies

$$
\left|x_{1}\right| \leq \alpha_{1}\left(e^{-1} \alpha_{2}\left(\left|x_{0}\right|\right)\right)+\gamma_{1}\left(\min _{w \in \mathbf{W}}\left\{|w|: f\left(x_{0}, u_{0}, w\right)=x_{1}\right\}\right),
$$


Now for system (88) with arbitrary initial condition $\zeta_{0}, \hat{\eta}_{0} \in \mathbf{R}$, we have

$$
\begin{aligned}
\left|x_{1}\right| & \leq \alpha_{1}\left(e^{-1}\left(\left|\zeta_{0}\right|+\alpha_{2}\left(\left|x_{0}\right|\right)\right)\right)+\gamma_{1}\left(\max \left\{a \hat{\eta}_{0}, \min _{w \in \mathbf{W}}\left\{|w|: f\left(x_{0}, u_{0}, w\right)=x_{1}\right\}\right\}\right) \\
& =\alpha_{1}\left(\zeta_{1}\right)+\gamma_{1}\left(\hat{\eta}_{1}\right) .
\end{aligned}
$$

Thus (89) holds for $k=1$.

The consequent control is chosen as

$$
u_{k}=\mathbf{u}^{1}\left(x_{k}, \zeta_{k}, \hat{\eta}_{k}\right), \quad k \geq 1
$$

where $\mathbf{u}^{1}(x, \zeta, \hat{\eta})$ can be obtained by solving the SFULIB problem for the auxiliary system

$$
\left\{\begin{aligned}
x_{k+1} & =f\left(x_{k}, u_{k}, w_{k}\right) \\
\zeta_{k+1} & =e^{-1} \zeta_{k} \\
\hat{\eta}_{k+1} & =\max \left\{a\left|\hat{\eta}_{k}\right|, \min _{w \in \mathbf{W}}\left\{|w|: f\left(x_{k}, u_{k}, w\right)=x_{k+1}\right\}\right\} \\
z_{k} & =\left|x_{k}\right|-\alpha_{1}\left(\zeta_{k}\right)-\gamma_{1}\left(\hat{\eta}_{k}\right)
\end{aligned}\right.
$$

with

$$
\hat{B}_{0}^{1}=\left\{\left(\begin{array}{c}
x_{0} \\
\zeta_{0} \\
\hat{\eta}_{0}
\end{array}\right):\left|x_{0}\right|-\alpha_{1}\left(\zeta_{0}\right)-\gamma_{1}\left(\hat{\eta}_{0}\right) \leq 0\right\}, \quad \lambda=0 .
$$

This will guarantee that (89) holds for all $k \geq 1$.

Though the closed-loop ISS property can be achieved, it is hard to obtain a clear result on what the achieved transient bound and asymptotic gain (in the ISS property (93),(91),(92)) are as a function of the gains used in the controllers. It is even harder to present the results on $\mathrm{SF} \star$ Problem in a unified manner. This is a reason why we did not present the results in this way.

\subsection{Computational Complexity}

The controller design methods proposed in this paper are expressed in terms of dynamic programming equations (or inequalities). If a given dynamic programming equation (or inequality) has a solution (satisfying some mild technical conditions), then the corresponding synthesis problem is solvable. It is well known that explicit solutions for dynamic programming equations are not generally available and approximate or numerical methods are required. Especially, in the measurement feedback case, numerical methods can only be used when the set-valued observer is finite dimensional (e.g. interval, sphere). Otherwise, approximate solutions have to be used. One possible way to find approximate solutions for dynamic programming equations (or inequalities) is using the idea of relaxed dynamic programming [17].

In the unified synthesis approach we provided in this paper, additional dimensions for the dynamic programming problem are introduced in order to achieve the desired decay rate and asymptotic gain. This further increases the computation burden. However, the computational complexity is due to the nature of the generality of the problem. For some particular problem, it may be possible to reduce the dimension of the problem by introducing dynamics for the value function, like the Lyapunov function for the input-tostate dynamical stability introduced by Grune [9]. 


\section{Conclusion}

In this paper, we first provided a unified definition of different ISS-like properties, then we considered the quantitative synthesis of ISS-like properties where the disturbances gain and the transient bound are prescribed. The synthesis problems are shown to be equivalent to an auxiliary synthesis problem of $l^{\infty}$ bounded robustness considered in [12]. It turns out that the design methods provided in $[11,12]$ is a powerful tool that can be applied to the synthesis of different ISS-like properties for the plant dynamics. Both the state feedback synthesis and measurement feedback synthesis problems can be solved using dynamic programming techniques.

The approach in this paper provides a link between ISS synthesis and $H^{\infty}$ synthesis literature $[11,12]$ but there is a lot of open questions left for future research. Further research work include, for example, the synthesis problems to achieve the closed-loop ISS-like properties, the synthesis problems to achieve optimal/suboptimal gains, and the reduction of the computation complexity, etc.

\section{References}

[1] D. Angeli, A Lyapunov approach to incremental stability properties, IEEE Trans. Aut. Contr., 47, 410-422, 2002.

[2] D. Angeli, E.D. Sontag and Y. Wang, A characterization of integral input-to-state stability, IEEE Trans. Aut. Control, 45, 1082-1097, 2000.

[3] A. Isidori and A. Astolfi, Disturbance attenuation and $H_{\infty}$-control via measurement feedback, IEEE Transactions on Automatic Control, 37, 1283-1293, 1992.

[4] T. Basar and P. Bernhard, " $H^{\infty}$-Optimal Control and Related Minimax Design Problems: A Dynamic Game Approach", 2nd Ed., Birkhauser, Boston, 1995.

[5] M.A. Dahleh and I.J. Diaz-Bobillo, "Control of Uncertain Systems: A Linear Programming Approach", Prentice-Hall, Englewood Cliffs, NJ, 1995.

[6] N. Elia and M.A. Dahleh, Minimization of the worst case peak-to-peak gain via dynamic programming: state feedback case, IEEE Trans. Aut. Control, 45(4), 687-701, 2000 .

[7] I.J. Fialho and T.T. Georgiou, Worst case analysis of nonlinear systems, IEEE Trans. Aut. Control, 44(6), 1180-1196, 1999.

[8] M. Green and D. Limebeer, "Linear Robust Control", Prentice Hall, Englewood Cliffs, NJ. 1995.

[9] L. Grune, Input-to-state dynamical stability and its Laypunov function charactrization, IEEE Trans. Aut. Control, 47(9), 1499-1504, 2002. 
[10] L. Grune, E.D. Sontag and F.R. Wirth, Asymptotic stability equals exponential stability, and ISS equals finite energy gain - if you twist your eyes, Systems \& Control Letters, 38, 127-134, 1999.

[11] J.W. Helton and M.R. James, "Extending $H^{\infty}$ Control to Nonlinear Systems: Control of Systems to Achieve Performance Objectives", Advances in Design and Control, SIAM, 1999.

[12] S. Huang and M.R. James, $l^{\infty}$-bounded robustness for nonlinear systems: analysis and synthesis, IEEE Trans. Aut. Control, 48(11), 1875-1891, 2003.

[13] Z.P. Jiang, A.R. Teel and L.Praly, Small-gain theorem for ISS systems and applications, Mathematics of Control, Signals, and Systems, 7, 95-120, 1994.

[14] Z.P. Jiang and Y. Wang, Input-to-state stability for discrete-time nonlinear systems, Automatica, 37, 857-869, 2001.

[15] H. J. Kushner and P. Dupuis, "Numerical Methods for Stochastic Control Problems in Continuous Time", Springer Verlag, 2001.

[16] M. Krichman, E.D. Sontag and Y. Wang, Input-output-to-state stability, SIAM J. Control and Optimization, 39(6), 1874-1928, 2001.

[17] B. Lincoln and A. Rantzer, Suboptimal dynamic programming with error bounds, Proceedings of the 41st Conference on Decision and Control, December 2002.

[18] D. Liberzon, E.D. Sontag and Y. Wang, Universal construction of feedback laws achieving ISS and integral-ISS disturbance attenuation, Systems \& Control Letters, 46, 111-127, 2002.

[19] E.D. Sontag, Smooth stabilization implies coprime factorization, IEEE Trans. Aut. Control, 34(4), 435-443, 1989.

[20] E.D. Sontag, Comments on integral variants of ISS, Systems \& Control Letters, 34, 93-100, 1998.

[21] E.D. Sontag, The ISS philosophy as a unifying framework for stability-like behavior, Nonlinear Control in the Year 2000 (Volume 2) (Lecture Notes in Control and Information Sciences, A. Isidori, F. Lamnabhi-Lagarrigue, and W. Respondek, eds.), Springer-Verlag, Berlin, 2000, pp. 443-468.

[22] J.S. Shamma and K.-Y. Tu, Set-valued observers and optimal disturbance rejection, IEEE Trans. Aut. Control, 42(2), 253-264, 1999.

[23] E.D. Sontag and Y. Wang, On characterizations of input to state stability property, Systems \& Control Letters, 24, 351-359, 1995.

[24] E.D. Sontag and Y. Wang, New characterizations of input to state stability property, IEEE Trans. Aut. Control, 41, 1283-1294, 1996. 
[25] E.D. Sontag and Y. Wang, Notions of input to output stability, Systems \& Control Letters, 38, 235-248, 1999.

[26] A.J. van der Schaft, " $L_{2}$-Gain and Passivity Techniques in Nonlinear Control", Springer Verlag, 1996.

[27] J.C. Willems, Dissipative dynamical systems - Part I: general theory, Archive of Rational Mechanics and Analysis, 45, 321-351, 1972. 